\title{
Non-Abelian gauged fracton matter field theory: Sigma models, superfluids, and vortices
}

\author{
Juven Wang $\mathbb{1}^{1,2, *}$ and Shing-Tung Yau ${ }^{1,3,4, \dagger}$ \\ ${ }^{1}$ Center of Mathematical Sciences and Applications, Harvard University, Cambridge, Massachusetts 02138, USA \\ ${ }^{2}$ School of Natural Sciences, Institute for Advanced Study, Einstein Drive, Princeton, New Jersey 08540, USA \\ ${ }^{3}$ Department of Mathematics, Harvard University, Cambridge, Massachusetts 02138, USA \\ ${ }^{4}$ Department of Physics, Harvard University, Cambridge, Massachusetts 02138, USA
}

(Received 31 December 2019; accepted 21 October 2020; published 12 November 2020)

\begin{abstract}
By gauging a higher-moment polynomial degree global symmetry and a discrete charge conjugation (i.e., particle-hole) symmetry coupled to matter fields (two symmetries mutually noncommutative), we derive a class of higher-rank tensor non-Abelian gauge field theory with dynamically gauged fractonic matter fields: Non-Abelian gauged fractons interact with a hybrid class of higher-rank (symmetric or generic nonsymmetric) tensor gauge theory and antisymmetric tensor topological field theory, generalizing work by Wang and $\mathrm{Xu}$ (arXiv:1909.13879) and by Wang, Xu, and Yau (arXiv:1911.01804). We also apply a quantum phase transition similar to that between insulator and superfluid/superconductivity [U(1) symmetry disordered phase described by a topological gauge theory or a disordered sigma model vs U(1) global/gauge symmetry-breaking ordered phase described by a sigma model with a U(1) target space underlying Goldstone modes]: We can regard our tensor gauge theories as disordered phases, and we transition to their ordered phases by deriving sigma models in continuum field theories. While one low energy theory is captured by degrees of freedom of rotor or scalar modes, another side of low energy theory has vortices and superfluids; we explore non-Abelian vortices (two types of vortices mutually interacting noncommutatively beyond an ordinary group structure) and their Cauchy-Riemann relation.
\end{abstract}

DOI: 10.1103/PhysRevResearch.2.043219

\section{INTRODUCTION AND OVERVIEW OF PREVIOUS WORKS}

Fracton order (see a recent review [1] in condensed matter) concerns conservation laws imposed on the energetic excitations (such that the particle excitations are known as fractons) of quantum systems which have significant restrictions on their mobility:

(1) Excitations cannot move without creating additional excitations (commonly known as fractons).

(2) Excitations can only move in certain subdimensional or subsystem directions (for zero-dimensional excitations known as subdimensional particles).

The origins of such constraints are conservation laws from conserved quantities of higher moments, including dipole moments [2] (relevant for a vector global symmetry in field theory [3,4]), quadrupole moments, generalized multipole moments (relevant for the so-called polynomial global symmetry [4-6] or the polynomial shift symmetries [7] in field

\footnotetext{
*Corresponding author: jw@ cmsa.fas.harvard.edu; http://sns.ias.edu/ juven/

†yau@math.harvard.edu
}

Published by the American Physical Society under the terms of the Creative Commons Attribution 4.0 International license. Further distribution of this work must maintain attribution to the author(s) and the published article's title, journal citation, and DOI. theory), etc. The composite excitation of each mobilityrestricted excitations are, however, mobile. The mobility constraint of fracton phases is also related to quantum glassy dynamics $[8,9]$. Following the previous work of Refs. [4,6], motivated by the fracton order in condensed matter [1], we continue extending and developing this framework by including the dynamically gauged matter fields in the higher-rank tensor gauge theory in a $d+1$-dimensional spacetime (e.g., $d+1 \mathrm{~d}$, over a flat spacetime manifold $M^{d+1}$, and we should focus on Cartesian coordinates $\left.\mathbb{R}^{d+1}\right) .{ }^{1}$ The important ingredient in our present work is that the gauge structure can be noncommutative (i.e., so-called non-Abelian), while still coupling to the matter fields; thus a partial goal of our present work is to derive a non-Abelian tensor gauged fracton field theory (that has gauge interactions also coupling with gauged matter fields).

The field-theoretic models given in Refs. [4,6] offer some unified features that we may summarize via examples that also connect to the literature:

(1) An ungauged matter field theory of higher-moment global symmetry without gauge fields (e.g., an ungauged abelian theory with a degree- 0 ordinary symmetry that encodes Schrödinger [10] or Klein-Gordon type field theory

\footnotetext{
${ }^{1}$ In this article, we follow the notations and definitions given in Refs. [4,6]. We attempt to be succinct in this article, thus we will directly guide the readers to refer the sections/materials derived in Refs. $[4,6]$.
} 
$[11,12]$ (see Sec. II A), with a degree-1 polynomial symmetry pioneered in Pretko's work [2] (see Sec. IIB) and its general higher-moment degree- $(m-1)$ polynomial general- ization [5,6] (see Sec. II C)): For example, there is a field theory captured by the Lagrangian term with a covariant derivate term $\left[P_{i_{1}, \ldots, i_{m}}\left(\Phi, \ldots, \partial^{m} \Phi\right)\right]$ or $P_{J}$ given in Secs. 2.1 and 3.1 of Ref. [6]. The schematic path integrals $\mathbf{Z}$ are

$$
\mathbf{Z}=\int[\mathcal{D} \Phi]\left[\mathcal{D} \Phi^{\dagger}\right] \exp \left(i \int_{M^{d+1}} d^{d+1} x\left(P_{i_{1}, \ldots, i_{m}}\left(\Phi, \ldots, \partial^{m} \Phi\right)\right)\left(P^{i_{1}, \ldots, i_{m}}\left(\Phi^{\dagger}, \ldots, \partial^{m} \Phi^{\dagger}\right)\right)\right)
$$

The dynamical complex scalar fields

$$
\Phi:=\Phi(x)=\Phi(\vec{x}, t) \quad \text { and } \quad \Phi^{\dagger}=\Phi^{\dagger}(x)=\Phi^{\dagger}(\vec{x}, t) \in \mathbb{C}
$$

are summed over in a schematic path integral. The Lagrangian term and $\mathbf{Z}$ are invariant under the global symmetry transformation.

(2) A pure Abelian or non-Abelian higher-rank tensor gauge theory (without coupling to gauged matter field):
The Abelian case is widely studied in various works in condensed matter literature; see Refs. [13-17].

But a non-Abelian version of fractonic theory is not much explored. Recent progress on non-Abelian fracton orders from Refs. [18-20] are mostly built from lattice models with a discrete gauge group (or a discrete gauge structure in general).

We will take an alternative route to non-Abelian fracton via the field theory. A rank-2 non-Abelian higher-rank tensor gauge theory with a continuous gauge structure is proposed in Ref. [4]. The most general form of rank- $m$ non-Abelian higher-rank tensor gauge theory is given by a schematic path integral in Ref. [6]'s Sec. 2.1:

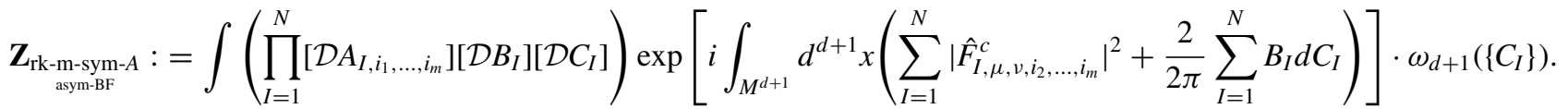

We can covert the cocycle $\omega_{d+1} \in \mathrm{H}^{d+1}\left(\left(\mathbb{Z}_{2}^{C}\right)^{N}, \mathbb{R} / \mathbb{Z}\right)$ as a group cohomology datum [21]tothe continuum topological quantum field theory (TQFT) formulation of discrete gauge theory (see Refs. [22-25] and the overview [4]). The $I$ is an index for specifying the different copies/layers of tensor gauge theories; the cocycle $\omega_{d+1}$ couples different copies/layers of tensor gauge theories together. Thus the cocycle $\omega_{d+1}$ gives rise to the interlayer interaction effects. The index $I$ may be neglected for simplicity below. The real-valued Abelian gauge field strength $F_{\mu, v, i_{2}, \ldots, i_{m}} \in \mathbb{R}$ is promoted into a complexvalued non-Abelian gauge field strength $\hat{F}_{\mu, v, i_{2}, \ldots, i_{m}}^{c} \in \mathbb{C}$ after gauging a discrete charge conjugation $\mathbb{Z}_{2}^{C}$ (i.e., particle-hole) symmetry $[4,6]$ :

$$
\begin{aligned}
\hat{F}_{\mu, \nu, i_{2}, \ldots, i_{m}}^{c} & :=D_{\mu}^{c} A_{v, i_{2}, \ldots, i_{m}}-D_{\nu}^{c} A_{\mu, i_{2}, \ldots, i_{m}} \\
& :=\left(\partial_{\mu}-i g_{c} C_{\mu}\right) A_{\nu, i_{2}, \ldots, i_{m}}-\left(\partial_{\nu}-i g_{c} C_{\nu}\right) A_{\mu, i_{2}, \ldots, i_{m}},
\end{aligned}
$$

while $\left|\hat{F}_{\mu, v, i_{2}, \ldots, i_{m}}^{c}\right|^{2}:=\hat{F}_{\mu, \nu, i_{2}, \ldots, i_{m}}^{c} \hat{F}^{\dagger c \mu, \nu, i_{2}, \ldots, i_{m}}$. Here are the field contents:

(a) The $A \in \mathbb{R}$ can be chosen to be a fully symmetric rank$m$ real-valued tensor gauge field.

(b) The $B \in \mathbb{R}$ is a $(d-1)$ th $\mathbb{Z}_{2}$-cohomology class in terms of $\mathbb{Z}_{2}$-discrete gauge theory, or in the continuum formulated as a $(d-1)$-form [an antisymmetric rank- $(d-1)$ tensor] real-valued gauge field. The $B$ plays the role of a Lagrangian multiplier to set $C$ to be flat.

(c) The $C \in \mathbb{R}$ is a $\mathbb{Z}_{2}$-cohomology class in terms of $\mathbb{Z}_{2}$ discrete gauge theory, or in the continuum formulated as a 1-form (a rank-1 tensor) real-valued gauge field.
(3) An Abelian gauge theory coupling to gauged matter field: This was pioneered by Pretko [2] for the rank-2 tensor fields, while we can use the most general form for the rank- $m$ tensor gauge field $A=A_{i_{1}, i_{2}, \ldots, i_{m}}$ given in Ref. [6], Sec. 2.1's and Sec. 3.1's schematic path integral:

$$
\begin{aligned}
\mathbf{Z}_{\mathrm{rk}-2 \text {-sym- } \Phi}= & \int[\mathcal{D} A][\mathcal{D} \Phi]\left[\mathcal{D} \Phi^{\dagger}\right] \exp \left(i \int_{M^{d+1}} d^{d+1} x\right. \\
& \left.\times\left(\left|F_{\mu, v, i_{2}, \ldots, i_{m}}\right|^{2}+\left|D^{A}[\{\Phi\}]\right|^{2}+V\left(|\Phi|^{2}\right)\right)\right),
\end{aligned}
$$

$\left|D^{A}[\{\Phi\}]\right|^{2}:=|R|^{2}:=(R)\left(R^{\dagger}\right)=(P-i g A \mathcal{Q})\left(P^{\dagger}+i g A \mathcal{Q}^{\dagger}\right)$.

The $D^{A}[\{\Phi\}]:=R \equiv P-i g A \mathcal{Q}$ is defined in Sec. 3.1 of Ref. [6]. Here $P$ and $\mathcal{Q}$ are polynomials of $\Phi$ and its differential of $\partial^{\ell} \Phi$ for some power of $\ell$. Here $P$ and $\mathcal{Q}$ are uniquely determined by the polynomial $Q(x)$ in the higher-moment global symmetry

$$
\begin{aligned}
\Phi_{I} & \rightarrow e^{i Q_{I}(x)} \Phi_{I} \\
& :=e^{i\left(\Lambda_{I ; i_{1}, \ldots, i_{m-1}} x_{i_{1}} \ldots x_{i_{m-1}}+\cdots+\Lambda_{I ; i, j} x_{i} x_{j}+\Lambda_{I ; i} x_{i}+\Lambda_{I ; 0}\right)} \Phi_{I},
\end{aligned}
$$

shown in Ref. [6]. We denote such a polynomial symmetry as $\mathrm{U}(1)_{\text {poly }}$ following [6]; see a review in Sec. II C 1.

What other topics have not yet been covered in the literature but should be formulated? We will focus on these two open issues:

(1) A non-Abelian gauge theory coupling to gauged matter field: 
Previous works only explored the Abelian gauged matter theory, or the non-Abelian gauge theory without coupling to (fractonic) matter fields [4,6]. In Sec II, we provide a systematic framework for non-Abelian gauged fractonic matter field theories.

In order to facilitate such a non-Abelian gauged matter formulation, we sometimes trade a single complex component $\Phi=\Phi_{\mathrm{Re}}+i \Phi_{\mathrm{Im}} \in \mathbb{C}$ into two real components $\left(\begin{array}{c}\Phi_{\mathrm{Re}} \in \mathbb{R} \\ \Phi_{\mathrm{Im}} \in \mathbb{R}\end{array}\right)$. For the $U(1)$ polynomial symmetry viewpoint, $\Phi \in \mathbb{C}$ is more natural. The $\mathbb{Z}_{2}^{C}$ (particle-hole or particle-antiparticle symmetry) transformation acts on $\Phi \rightarrow \Phi^{\dagger}$ in the complex U(1) basis, but the $\mathbb{Z}_{2}^{C}$ acts on the two-component field naturally as

$$
\left(\begin{array}{c}
\Phi_{\mathrm{Re}} \\
\Phi_{\mathrm{Im}}
\end{array}\right) \rightarrow\left(\begin{array}{c}
\Phi_{\mathrm{Re}} \\
-\Phi_{\mathrm{Im}}
\end{array}\right)
$$

To introduce the non-Abelian gauge coupling to the matter fields, we will need to introduce several types of gauge derivatives. ${ }^{2}$ For example, even for the simplest degree- 0 polynomial symmetry with a rank-1 tensor gauge field (1form gauge field $A_{\mu}$ ), we require

$$
\begin{aligned}
D_{\mu}^{c, \operatorname{Im}} \Phi:= & \partial_{\mu} \Phi-i g_{c} C_{\mu} \Phi_{\mathrm{Im}}, \\
D_{\mu}^{A} \Phi:= & \left(\partial_{\mu}-i g A_{\mu}\right) \Phi=\left(\partial_{\mu} \Phi_{\mathrm{Re}}+g A_{\mu} \Phi_{\mathrm{Im}}\right) \\
& +i\left(\partial_{\mu} \Phi_{\mathrm{Im}}-g A_{\mu} \Phi_{\mathrm{Re}}\right) \\
D_{\mu}^{A, c, \operatorname{Im}} \Phi:= & D_{\mu}^{A} \Phi-i g_{c} C_{\mu} \Phi_{\mathrm{Im}} \\
= & \left(\partial_{\mu}-i g A_{\mu}\right) \Phi-i g_{c} C_{\mu} \Phi_{\mathrm{Im}} \\
D_{\mu}^{c}:= & \left(\partial_{\mu}-i g_{c} C_{\mu, I}\right), \\
D_{\mu}^{c} A_{\nu, i_{2}, \ldots, i_{m}}:= & \left(\partial_{\mu}-i g_{c} C_{\mu}\right) A_{v, i_{2}, \ldots, i_{m}} .
\end{aligned}
$$

The first line $D_{\mu}^{c \text {,Im }}$ has the $C$ gauge field that only couples to the charged matter $\Phi_{\operatorname{Im}}$ (the imaginary component) under $\mathbb{Z}_{2}^{C}$. The second line $D_{\mu}^{A}$ is the gauge covariant derivative of 1-form gauge field $A_{\mu}$ after gauging the ordinary 0-form U(1) symmetry (the degree-0 polynomial symmetry). The third line $D_{\mu}^{A, c, \operatorname{Im}} \Phi$ shows the gauge derivative on $\Phi$ involving both the $\mathrm{U}(1) \rtimes \mathbb{Z}_{2}^{C}=\mathrm{O}(2)$ gauge fields. The fourth and the fifth lines show that for the fields charged under $\mathbb{Z}_{2}^{C}$ (e.g., the rank$m$ symmetric tensor $A_{v, i_{2}, \ldots, i_{m}} \rightarrow-A_{v, i_{2}, \ldots, i_{m}}$ is charged under $\mathbb{Z}_{2}^{C}$ ), then the gauge derivative is $D_{\mu}^{c}$. The $g_{c}$ is a $\mathbb{Z}_{2}^{C}$ gauge coupling denoted explicitly for convenience.

We will present explicit examples to gauge both highermoment and charge conjugation global symmetries including the matter in Sec. II.

(2) An alternative type of sigma model: We formulate an alternative type of sigma model that can move between the ordered and disordered phases of these higher-rank non-Abelian tensor field theories with fully gauged fractonic matter. ${ }^{3}$ Similar to the familiar quantum phase transition between insulator

\footnotetext{
${ }^{2}$ Note that some of such gauge derivatives are not gauge covariant under the gauge transformations, due to the non-Gaussian nature and higher-moment terms appearing already in the fractonic matter field theories. But we will be able to construct such types of gauge covariant terms and the gauge invariant Lagrangian in Sec. II.

${ }^{3}$ In terms of the old Landau-Ginzburg paradigm, this is related to the sigma model formulation of Landau-Ginzburg theory [26].
}

and superfluid/superconductivity [27-29] [U(1) symmetry disorder described by a topological gauge theory or a disordered sigma model vs U(1) global/gauge symmetry-breaking order described by a sigma model with a U(1) target space with Goldstone modes), we can regard our tensor gauge theory as a disordered phase, and we drive to its ordered phase by deriving a sigma model in terms of continuum field theory.

Very recently, the superfluid and vortices of an Abelian version of pure fractonic theories (without gauge fields) were studied in [30]. Two ingredients in our work, which are not present in Ref. [30], are the facts that we include the gauge field interactions (thus we include the additional long-range entanglements) and we also include the non-Abelian gaugematter interactions.

\section{FROM ABELIAN GAUGE FRACTONIC THEORIES TO NON-ABELIAN GAUGED FRACTONIC MATTER THEORIES}

\section{A. Degree-0 polynomial symmetry to Schrödinger or Klein-Gordon type field theory}

\section{Global-covariant 1-derivative}

Suppose we want to construct a field theory that preserves a degree-0 polynomial symmetry with a polynomial $Q(x)=$ $\Lambda_{0}$. Then a complex scalar field transforms as $\Phi:=\Phi(x)=$ $\Phi(\vec{x}, t) \in \mathbb{C}$,

$$
\Phi \rightarrow e^{i Q(x)} \Phi=e^{i \Lambda_{0}} \Phi
$$

while its logarithm transforms as

$$
\log \Phi \rightarrow \log \Phi+i \Lambda_{0}
$$

Taking the derivative $\partial_{x_{i}}:=\partial_{i}$ respect to coordinates on both sides, we can eliminate $\partial_{i} \Lambda_{0}$, thus we get an invariant term:

$$
\partial_{i} \log \Phi \rightarrow \partial_{i} \log \Phi
$$

This means $\partial_{i} \log \Phi$ is invariant under the global symmetry transformation. We can also define

$$
\partial_{i} \log \Phi:=\frac{P_{i}(\Phi, \partial \Phi)}{\Phi}=\frac{\partial_{i} \Phi}{\Phi} .
$$

Under $\Phi \rightarrow e^{i Q(x)} \Phi$, since $\partial_{i} \log \Phi$ is invariant and the denominator $\Phi \rightarrow e^{i Q(x)} \Phi$ is covariant, the numerator $P_{i}(\Phi, \partial \Phi)=\partial_{i} \Phi \rightarrow e^{i Q(x)} \partial_{i} \Phi$ is also covariant. Namely, both the numerator $P_{i}(\Phi, \partial \Phi)$ and $\Phi$ are global covariant under $\Phi \rightarrow e^{i Q(x)} \Phi$, in order to maintain $\partial_{i} \log \Phi$ invariant. Here $P_{i}(\Phi, \partial \Phi)$ denotes some functional $P_{i}$ that depends on field $\Phi$ or its derivative $\partial \Phi$.

For convenience, we will call such construction a globalcovariant 1-derivative,

$$
P_{i}(\Phi, \partial \Phi):=\partial_{i} \Phi
$$

to facilitate its further generalization later. We also construct a globally invariant Lagrangian

$$
\left|P_{i}\right|^{2}+V\left(|\Phi|^{2}\right)
$$


that contains a potential term $V\left(|\Phi|^{2}\right)$ and a kinetic term ${ }^{4}$ :

$$
\left|P_{i}\right|^{2}:=P_{i}(\Phi) P^{i}\left(\Phi^{\dagger}\right)=\partial_{i} \Phi \partial^{i} \Phi^{\dagger} .
$$

In this way, based on the systematic method of Ref. [6], we can rederive a Lagrangian formulation of the Schrödinger equation from 1925 [10] and Klein-Gordon theory from 1926 $[11,12]$ for complex scalar fields.

\section{Gauge-covariant 1-derivative}

To gauge a degree-0 polynomial symmetry, we rewrite $Q(x)$ as a local gauge parameter $\eta(x)$,

$$
\begin{aligned}
\Phi & \rightarrow e^{i \eta(x)} \Phi, \\
\partial_{i} \log \Phi & \rightarrow \partial_{i} \log \Phi+i \partial_{i} \eta(x) .
\end{aligned}
$$

Then $\partial_{i} \log \Phi$ is no longer an invariant term. This implies that we can write a gauge-covariant operator $D_{i}^{A}[\{\Phi\}]$ via combining $P_{i}$ and $A_{i}$ :

$$
\begin{aligned}
P_{i}(\Phi, \partial \Phi) & :=\partial_{i} \Phi \rightarrow e^{i \eta(x)}\left[P_{i}(\Phi, \partial \Phi)+i \partial_{i} \eta(x)\right], \\
A_{i} & \rightarrow A_{i}+\frac{1}{g} \partial_{i} \eta, \\
D_{i}^{A}[\{\Phi\}] & :=P_{i}(\Phi, \partial \Phi)-i g A_{i} \Phi=\partial_{i} \Phi-i g A_{i} \Phi, \\
D_{i}^{A}[\{\Phi\}] & \rightarrow e^{i \eta(x)} D_{i}^{A}[\{\Phi\}] .
\end{aligned}
$$

To obtain a gauge invariant term, we can pair the gaugecovariant operator with its complex conjugation to obtain a gauge invariant Lagrangian,

$$
\begin{aligned}
& \left|D_{i}^{A}[\{\Phi\}]\right|^{2}+V\left(|\Phi|^{2}\right) \\
& \quad=\left[\left(\partial_{i}-i g A_{i}\right) \Phi\right]\left[\left(\partial^{i}+i g A^{i}\right) \Phi^{\dagger}\right]+V\left(|\Phi|^{2}\right) .
\end{aligned}
$$

\section{Gauge-covariant non-Abelian rank-2 field strength}

Notice that in the pure matter theory, Eq. (2.6) without gauge fields, we already have a degree-0 U(1) global symmetry and a $\mathbb{Z}_{2}^{C}$ discrete charge conjugation (i.e., particle-hole) symmetry:

$$
\Phi \mapsto \Phi^{\dagger},
$$

which makes Eq. (2.6) invariant. It is easy to see that the symmetry group structure is a non-Abelian group

$$
\mathrm{U}(1) \rtimes \mathbb{Z}_{2}^{C}=\mathrm{SO}(2) \rtimes \mathbb{Z}_{2}^{C}=\mathrm{O}(2),
$$

which acts on the $\Phi$ noncommutatively:

$$
\begin{aligned}
& U_{\mathbb{Z}_{2}^{c}} U_{\mathrm{U}(1)} \Phi=U_{\mathbb{Z}_{2}^{c}}\left(e^{i \eta} \Phi\right)=e^{i \eta} \Phi^{\dagger}, \\
& U_{\mathrm{U}(1)} U_{\mathbb{Z}_{2}^{c}} \Phi=U_{\mathrm{U}(1)}\left(\Phi^{\dagger}\right)=e^{-i \eta} \Phi^{\dagger} .
\end{aligned}
$$

After we dynamically gauge the $\mathrm{U}(1)$ symmetry to obtain Eq. (2.14), we can still keep $\mathbb{Z}_{2}^{C}$ discrete charge conjugation (i.e., particle-hole) symmetry intact, which acts on gauge fields as

$$
A_{i} \mapsto-A_{i}, \quad \eta(x) \mapsto-\eta(x) .
$$

\footnotetext{
${ }^{4}$ The raising and the lowering indices are merely used for contractions and summation, e.g., we sum over $i$ indices in $\partial_{i} \Phi \partial^{i} \Phi^{\dagger}$. The conversion between the raising and lowering indices does not involve spacetime metrics; we only consider the flat spacetime.
}

If we fully gauge $\mathrm{U}(1) \rtimes \mathbb{Z}_{2}^{C}=\mathrm{O}(2)$, we get a non-Abelian $\mathrm{O}(2)$ gauge transformations which acts also on gauge fields noncommutatively:

$$
\begin{aligned}
& U_{\mathbb{Z}_{2}^{c}} U_{\mathrm{U}(1)} A_{j}=U_{\mathbb{Z}_{2}^{c}}\left(A_{j}+\frac{1}{g} \partial_{j} \eta\right)=-A_{j}+\frac{1}{g} \partial_{j} \eta . \\
& U_{\mathrm{U}(1)} U_{\mathbb{Z}_{2}^{c}} A_{j}=U_{\mathrm{U}(1)}\left(-A_{j}\right)=-A_{j}-\frac{1}{g} \partial_{j} \eta .
\end{aligned}
$$

By promoting the global $\mathbb{Z}_{2}^{C}$ to a local symmetry, we introduce a 1 -form $\mathbb{Z}_{2}^{C}$-gauge field $C$ coupling to the 0 -form symmetry $\mathbb{Z}_{2}^{C}$-charged object $A_{i}$ with a $g_{c}$ coupling. The $\mathbb{Z}_{2}^{C}$ local gauge transformation is

$$
A_{i} \rightarrow e^{i \gamma_{c}(x)} A_{i}, \quad C_{i} \rightarrow C_{i}+\frac{1}{g_{c}} \partial_{i} \gamma_{c}(x) .
$$

Note $A_{i_{1}} \in \mathbb{R}$ is real valued, so a generic $e^{i \gamma_{c}(x)}$ complexifies the $A_{i}$. Thus we restrict gauge transformation to be only $\mathbb{Z}_{2}^{C}$ gauged [not $\mathrm{U}(1)^{C}$ gauged]:

$$
e^{i \gamma_{c}(x)}:=(-1)^{\gamma_{c}^{\prime}(x)} \in\{ \pm 1\}, \quad \gamma_{c} \in \pi \mathbb{Z}, \gamma_{c}^{\prime} \in \mathbb{Z},
$$

so $\gamma_{c}^{\prime}(x)$ is an integer and $A_{i} \rightarrow \pm A_{i}$ stays real. In the continuum field theory, the restricted gauge transformation is done by coupling to a level-2 BF theory (the $\mathbb{Z}_{2}^{C}$-gauge theory) $[4,6]$. Thus $\gamma_{c}^{\prime}(x)$ jumps between even or odd integers in $\mathbb{Z}$, while the $\mathbb{Z}_{2}^{C}$-gauge transformation can be suitably formulated on a lattice. We can also directly express the above on a triangulable spacetime manifold or a simplicial complex.

Approach 1: Gauge 0-form $\mathbb{Z}_{2}^{C}$ symmetry of 1-form gauge field A. Following Ref. [4], we also define a covariant derivative with respect to $\mathbb{Z}_{2}^{C}$ :

$$
D_{i}^{c}:=\left(\partial_{i}-i g_{c} C_{i}\right) .
$$

We obtain a combined $\mathrm{O}(2)$ gauge transformation of $A_{j}$,

$$
\begin{aligned}
A_{j} & \rightarrow e^{i \gamma_{c}(x)} A_{j}+( \pm)^{\rtimes} \frac{1}{g}\left(D_{j}^{c}\right)[\eta(x)] \\
& :=\left\{\begin{array}{l}
V_{\mathbb{Z}_{2}} V_{\mathrm{U}(1)} A_{j}=e^{i \gamma_{c}(x)} A_{j}+\frac{1}{g} D_{j}^{c} \eta, \\
V_{\mathrm{U}(1)} V_{\mathbb{Z}_{2}} A_{j}=e^{i \gamma_{c}(x)}\left(A_{j}+\frac{1}{g} D_{j}^{c} \eta\right)
\end{array} .\right.
\end{aligned}
$$

We define an operation

$$
( \pm)^{\rtimes} \in\{+1,-1\}
$$

via the above Eq. (2.22). Only when we perform a $\mathbb{Z}_{2}^{C}$ first and then $\mathrm{U}(1)$ gauge transformation second, and when $e^{i \gamma_{c}(x)}=$ -1 , do we have $( \pm)^{\rtimes}=-1$; otherwise all the other cases $( \pm)^{\rtimes}=+1$. This factor $( \pm)^{\rtimes}$ also captures the non-Abelianness of the gauge structure.

References $[4,6]$ define a rank-2 non-Abelian field strength as

$$
\begin{aligned}
\hat{F}_{i_{1}, i_{2}}^{c}:= & D_{i_{1}}^{c} A_{i_{2}}-D_{i_{2}}^{c} A_{i_{1}}:=\left(\partial_{i_{1}}-i g_{c} C_{i_{1}}\right) A_{i_{2}} \\
& -\left(\partial_{i_{2}}-i g_{c} C_{i_{2}}\right) A_{i_{1}},
\end{aligned}
$$

with the locally flat $\mathbb{Z}_{2}^{C}$-gauge field $C$ imposed by the level2 BF theory $\frac{2}{2 \pi} \int B d C$. It can be shown that $\hat{F}_{i_{1}, i_{2}}^{c}$ is gaug covariant under the $\mathrm{O}(2)$ gauge transformation Eq. (2.22):

$$
\hat{F}_{i_{1}, i_{2}}^{c} \rightarrow e^{i \gamma_{c}(x)} \hat{F}_{i_{1}, i_{2}}^{c} .
$$


The above rank-2 field strength utilizes the viewpoint of gauging 0 -form $\mathbb{Z}_{2}^{C}$ symmetry of 1 -form gauge field $A$. However, because $\hat{F}_{i_{1}, i_{2}}^{c}$ is an $\mathrm{O}(2)$ field strength, we can write $\hat{F}_{i_{1}, i_{2}}^{c}$ in a conventional way as a $2 \times 2$ matrix like Yang and Mills [31] did. We will pursue this alternative way in the next paragraph.

Approach 2: Non-Abelian O(2) field strength. From the $\mathrm{U}(1)$ symmetry transformation, a single complex component

$$
\Phi=\Phi_{\operatorname{Re}}+i \Phi_{\operatorname{Im}} \in \mathbb{C}
$$

is a more natural view. From the $\mathrm{O}(2)=\mathrm{SO}(2) \times \mathbb{Z}_{2}^{C}$ view, the two-component real scalar field $\left(\Phi_{\mathrm{Re}} \in \mathbb{R}, \Phi_{\operatorname{Im}} \in \mathbb{R}\right)$ is natural such that the $U_{\mathrm{U}(1)}$ and $U_{\mathbb{Z}_{2}^{C}}$ symmetry transforms the two-component field as

$$
\begin{aligned}
U_{\mathrm{U}(1)} & :\left(\begin{array}{l}
\Phi_{\mathrm{Re}} \\
\Phi_{\mathrm{Im}}
\end{array}\right) \rightarrow\left(\begin{array}{cc}
\cos (\theta) & \sin (\theta) \\
-\sin (\theta) & \cos (\theta)
\end{array}\right)\left(\begin{array}{l}
\Phi_{\mathrm{Re}} \\
\Phi_{\mathrm{Im}}
\end{array}\right), \\
U_{\mathbb{Z}_{2}^{C}}: & \left(\begin{array}{l}
\Phi_{\mathrm{Re}} \\
\Phi_{\mathrm{Im}}
\end{array}\right) \rightarrow\left(\begin{array}{c}
\Phi_{\mathrm{Re}} \\
-\Phi_{\mathrm{Im}}
\end{array}\right) .
\end{aligned}
$$

Let the $A$ gauge field be the generator of $U_{\mathrm{U}(1)}$, and the $C$ gauge field be the generator of $U_{\mathbb{Z}_{2}^{C}}$. We can write down the non-Abelian $\mathrm{O}(2)$ gauge field $X$ and field strength $\hat{F}_{X}$ with a Lie algebra generator as ${ }^{5}$

$$
\begin{aligned}
X & =\left(\begin{array}{cc}
0 & A \\
-A & g_{c} C
\end{array}\right) \\
\hat{F}_{X} & =d X-i X X=d\left(\begin{array}{cc}
0 & A \\
-A & g_{c} C
\end{array}\right)-i\left(\begin{array}{cc}
0 & A \\
-A & g_{c} C
\end{array}\right)\left(\begin{array}{cc}
0 & A \\
-A & g_{c} C
\end{array}\right)=\left(\begin{array}{cc}
0 & d A \\
-d A & g_{c} d C
\end{array}\right)-i g_{c}\left(\begin{array}{cc}
0 & A C \\
A C & 0
\end{array}\right) \\
& =\left(\begin{array}{cc}
0 & d A-i g_{c} A C \\
-d A-i g_{c} A C & 0
\end{array}\right) .
\end{aligned}
$$

Here we use the fact that $d C$ is locally flat.

The Yang-Mills $\mathrm{O}(2)$ field strength kinetic term from $\hat{F}_{X}$ is proportional to

$$
\begin{aligned}
\operatorname{Tr}\left[\hat{F}_{X} \wedge \star \hat{F}_{X}^{\dagger}\right] & =\operatorname{Tr}\left[\left(\begin{array}{cc}
0 & \partial_{\mu} A_{v}-i g_{c} A_{\mu} C_{v} \\
-\partial_{\mu} A_{v}-i g_{c} A_{\mu} C_{v} & 0
\end{array}\right)\left(\begin{array}{cc}
0 & -\partial^{\mu} A^{v}+i g_{c} A^{\mu} C^{v} \\
\partial^{\mu} A^{v}+i g_{c} A^{\mu} C^{v} & 0
\end{array}\right)\right] d^{4} x \\
& =2\left(\left(\partial_{\mu} A_{v}\right)^{2}+g_{c}\left(A_{\mu} C_{v}\right)^{2}\right) d^{4} x
\end{aligned}
$$

In comparison, the rank-2 non-Abelian field strength $\hat{F}_{\mu \nu}^{c}$ defined in Ref. [6] and Eq. (2.23) outputs

$$
\begin{aligned}
\hat{F}_{\mu \nu}^{c} \hat{F}^{c \mu \nu \dagger} & =\left[\left(\partial_{\mu}-i g_{c} C_{\mu}\right) A_{\nu}-\left(\partial_{\nu}-i g_{c} C_{\nu}\right) A_{\mu}\right]\left[\left(\partial^{\mu}+i g_{c} C^{\mu}\right) A^{\nu}-\left(\partial^{\nu}+i g_{c} C^{\nu}\right) A^{\mu}\right] \\
& \propto \partial_{\mu} A_{\nu} \partial^{\mu} A^{\nu}-\left(g_{c}\right)^{2}\left(A_{\mu} C_{\nu}\right)^{2} .
\end{aligned}
$$

Thus two approaches agree on the Yang-Mills Lagrangian $\operatorname{Tr}\left[\hat{F}_{X} \wedge \star \hat{F}_{X}^{\dagger}\right] \sim \hat{F}_{\mu \nu}^{c} \hat{F}^{c \mu \nu \dagger}$, up to a normalization constant.

Gauge-covariant non-Abelian rank-2 field strength. Under the $\mathrm{O}(2)$ gauge transformation,

$$
A_{j} \rightarrow A_{j}^{\prime}=e^{i \gamma_{c}(x)} A_{j}+( \pm)^{\rtimes} \frac{1}{g}\left(D_{j}^{c}\right)[\eta(x)] \eta, \quad C_{j} \rightarrow C_{j}^{\prime}=C_{j}+\frac{1}{g_{c}} \partial_{j} \gamma_{c}(x),
$$

we can explicitly check that the non-Abelian rank-2 field strength $\hat{F}_{\mu \nu}^{c}$ is gauge covariant:

$$
\begin{aligned}
\hat{F}_{\mu \nu}^{c} & :=D_{\mu}^{c} A_{\nu}-D_{\nu}^{c} A_{\mu}:=\left(\partial_{\mu}-i g_{c} C_{\mu}\right) A_{\nu}-\left(\partial_{\nu}-i g_{c} C_{\nu}\right) A_{\mu} \\
& \rightarrow e^{i \gamma_{c}} \hat{F}_{\mu \nu}^{c}+\frac{1}{g}\left(D_{\mu}^{c} D_{\nu}^{c}-D_{\nu}^{c} D_{\mu}^{c}\right) \eta_{v}=e^{i \gamma_{c}} \hat{F}_{\mu \nu}^{c},
\end{aligned}
$$

where we list the leading order terms, omitting the potentially higher power of $\eta$ and $\gamma_{c}$ terms. Note that

\footnotetext{
${ }^{5}$ Readers may wonder whether the $\mathrm{O}(2)$ Lie algebra generator needs to be traceless. There are, however, two facts:

(i) It is known that Lie algebra generators of a semisimple Lie algebra must be traceless. A Lie algebra is semisimple if it is a direct sum of simple Lie algebras, i.e., non-Abelian Lie algebras $\mathfrak{g}$ whose only ideals are 0 and $\mathfrak{g}$ itself. However, a one-dimensional Lie algebra (which is necessarily Abelian) is by definition not considered a simple Lie algebra, although such an algebra has no nontrivial ideals. Thus, onedimensional algebras are not allowed as summands in a semisimple Lie algebra.

(ii) The $C$ is a discrete $\mathbb{Z}_{2}^{C} 1$-form gauge field so $d C$ is locally flat. Later on we need to impose the condition to show gauge covariance of field strength. (In general we do not have to impose equations of motion to show gauge invariance, although, in the case with the $\mathbb{Z}_{2}^{C}$-gauge field $C$, we do require its locally flatness for gauge covariance of $\hat{F}_{i_{1}, i_{2}}^{c}$ or $\hat{F}_{X}$.)
} 


$$
\begin{aligned}
\left(D_{\mu}^{c} D_{\nu}^{c}-D_{\nu}^{c} D_{\mu}^{c}\right) & =\left(\partial_{\mu}-i g_{c} C_{\mu}\right)\left(\partial_{\nu}-i g_{c} C_{\nu}\right)-\left(\partial_{\nu}-i g_{c} C_{\nu}\right)\left(\partial_{\mu}-i g_{c} C_{\mu}\right) \\
& =\left(\partial_{\mu} \partial_{\nu}-\partial_{\nu} \partial_{\mu}\right)-i g_{c}\left(\partial_{\mu} C_{\nu}-\partial_{\nu} C_{\mu}\right)-i g_{c}\left(C_{\nu} \partial_{\mu}-C_{\mu} \partial_{\nu}\right)-i g_{c}\left(C_{\mu} \partial_{\nu}-C_{\nu} \partial_{\mu}\right)-g_{c}^{2}\left(C_{\mu} C_{\nu}-C_{\nu} C_{\mu}\right) \\
& =-i g_{c}\left(\partial_{\mu} C_{\nu}-\partial_{\nu} C_{\mu}\right)=-i g_{c}(d C)_{\mu \nu}=0,
\end{aligned}
$$

where we need to impose the locally flat condition for the $\mathbb{Z}_{2}^{C}$ gauge field in the last equality. ${ }^{6}$ Thus the gauge covariance is true since we show $\left(D_{\mu}^{c} D_{v}^{c}-D_{v}^{c} D_{\mu}^{c}\right)=0$.

\section{Non-Abelian $O(2)$ gauged matter: Polynomial invariant vs Yang-Mills method}

Previous work [4,6] does not couple non-Abelian gauge fields to matter field. In this work, we propose a systematic method to generate non-Abelian gauged matter theories.

Approach 1: Polynomial invariant method-Covariant derivative on the log as an invariant. For any give complex field $\mathfrak{N} \in \mathbb{C}$, such that $\mathfrak{N}=\mathfrak{N}_{\operatorname{Re}}+i \mathfrak{N}_{\mathrm{Im}}$, where the imaginary $\mathfrak{N}_{\text {Im }} \rightarrow-\mathfrak{N}_{\text {Im }}$ is charged under $\mathbb{Z}_{2}^{C}$ symmetry, we define a derivative

$D_{\mu}^{c, \operatorname{Im}} \mathfrak{N}:=\partial_{\mu} \mathfrak{N}_{\mathrm{Re}}+i D_{\mu}^{c} \mathfrak{N}_{\mathrm{Im}} \equiv \partial_{\mu} \mathfrak{N}_{\mathrm{Re}}+i\left(\partial_{\mu}-i g_{c} C_{\mu}\right) \mathfrak{N}_{\mathrm{Im}}$.

For example, for the complex scalar field $\Phi=\Phi_{\mathrm{Re}}+i \Phi_{\mathrm{Im}} \in$ $\mathbb{C}$, with the real component $\Phi_{\mathrm{Re}} \in \mathbb{R}$ and imaginary compo- nent $\Phi_{\operatorname{Im}} \in \mathbb{R}$,

$D_{\mu}^{c, \operatorname{Im}} \Phi:=\partial_{\mu} \Phi_{\mathrm{Re}}+i D_{\mu}^{c} \Phi_{\mathrm{Im}} \equiv \partial_{\mu} \Phi_{\mathrm{Re}}+i\left(\partial_{\mu}-i g_{c} C_{\mu}\right) \Phi_{\mathrm{Im}}$.

Following Eqs. (2.8) and (2.9), in order to find an $\mathrm{O}(2)$ gauge covariant derivative, we aim to first design an invariant term under gauge transformations. First, we see that $D_{\mu}^{c, \operatorname{Im}} \log \Phi$ is not invariant under a $\mathrm{U}(1)$ part of $\mathrm{O}(2)$ gauge transformation $\Phi \rightarrow e^{i \eta(x)} \Phi:$

$$
\begin{aligned}
& D_{\mu}^{c, \operatorname{Im}} \log \Phi \rightarrow D_{\mu}^{c, \operatorname{Im}} \log \Phi+D_{\mu}^{c, \operatorname{Im}}[i \eta(x)] \\
& \quad=\frac{D_{\mu}^{c, \operatorname{Im}} \Phi+D_{\mu}^{c, \operatorname{Im}}[i \eta(x)] \Phi}{\Phi}=\frac{D_{\mu}^{c, \operatorname{Im}} \Phi+i\left(D_{\mu}^{c} \eta\right) \Phi}{\Phi} .
\end{aligned}
$$

Here $\eta(x) \in \mathbb{R}$ is part of the complex phase of $e^{i \eta(x)} \Phi$, thus $D_{\mu}^{c, \operatorname{Im}}[i \eta(x)]=i\left(D_{\mu}^{c} \eta\right)$. Now based on the same trick in Eq. (2.12), we can absorb the U(1) gauge transformation by introducing the 1 -form $A$ gauge field. The $A$ transforms to $A^{\prime}$ under the $\mathrm{U}(1)$ part of Eq. (2.22):

$$
\begin{aligned}
A_{j} & \rightarrow A_{j}^{\prime}=A_{j}+\frac{1}{g} D_{j}^{c} \eta, \\
D_{\mu}^{A, c, \operatorname{Im}} \log \Phi=\frac{D_{\mu}^{A, c, \operatorname{Im}} \Phi}{\Phi} & \rightarrow D_{\mu}^{A^{\prime}, c, \operatorname{Im}} \log \Phi+D_{\mu}^{A^{\prime}, c, \operatorname{Im}}[i \eta(x)]=\frac{D_{\mu}^{A^{\prime}, c, \operatorname{Im}} \Phi+i\left(D_{\mu}^{c} \eta\right) \Phi}{\Phi} \\
& =\frac{\left[\partial_{\mu}-i g\left(A_{j}+\frac{1}{g} D_{j}^{c} \eta\right)\right] \Phi-i g_{c} C_{\mu} \Phi_{\operatorname{Im}}+i\left(D_{\mu}^{c} \eta\right) \Phi}{\Phi}=\frac{D_{\mu}^{A, c, \operatorname{Im}} \Phi}{\Phi} .
\end{aligned}
$$

Here $D_{\mu}^{A^{\prime}, c, \operatorname{Im}}[i \eta(x)]=i\left(D_{\mu}^{c} \eta\right)$ because $\eta$ is not charged under $\mathrm{U}(1)$ symmetry but only the $\mathrm{U}(1)$ gauge parameter of $A$ itself. The above shows that $D_{\mu}^{A, c, \operatorname{Im}} \log \Phi=\frac{D_{\mu}^{A, c, \operatorname{Im}} \Phi}{\Phi}$ is a gauge invariant quantity under $\mathrm{U}(1)$ gauge transformation. We can show that it is also gauge invariant quantity under the full $\mathrm{O}(2)$ gauge transformation [including Eq. (2.22) and the definition of $( \pm)^{\rtimes} \in\{+1,-1\}$ around Eq. (2.22)]:

$$
\begin{aligned}
& A_{j} \rightarrow A_{j}^{\prime}=e^{i \gamma_{c}(x)} A_{j}+( \pm)^{\rtimes} \frac{1}{g}\left(D_{j}^{c}\right)[\eta(x)] \eta, \\
& C_{j} \rightarrow C_{j}^{\prime}=C_{j}+\frac{1}{g_{c}} \partial_{j} \gamma_{c}(x), \\
& \Phi \rightarrow e^{( \pm)^{\rtimes} i \eta(x)} \Phi_{c}=e^{( \pm)^{\rtimes} i \eta(x)}\left(\Phi_{\mathrm{Re}}+i e^{i \gamma_{c}(x)} \Phi_{\mathrm{Im}}\right)= \begin{cases}e^{i \eta(x)} \Phi & \text { if } \gamma_{c} \in \pi \mathbb{Z}_{\mathrm{even}}, \\
e^{( \pm)^{\rtimes} i \eta(x)} \Phi^{\dagger} & \text { if } \gamma_{c} \in \pi \mathbb{Z}_{\text {odd }} .\end{cases}
\end{aligned}
$$

Let us focus on the case $\Phi \rightarrow e^{i \eta(x)} \Phi_{c}$ first. We have

$$
\begin{aligned}
& D_{\mu}^{A, c, \operatorname{Im}} \log \Phi=\frac{D_{\mu}^{A, c, \operatorname{Im}} \Phi}{\Phi} \rightarrow D_{\mu}^{A^{\prime}, c^{\prime}, \operatorname{Im}} \log \Phi_{c}+D_{\mu}^{A^{\prime}, c^{\prime}, \operatorname{Im}}[i \eta(x)]=\frac{D_{\mu}^{A^{\prime}, c^{\prime}, \operatorname{Im}} \Phi_{c}+i\left(D_{\mu}^{c^{\prime}} \eta\right) \Phi_{c}}{\Phi_{c}} \\
& =\frac{\left[\partial_{\mu}-i g\left(A_{j}+\frac{1}{g} D_{j}^{c^{\prime}} \eta\right)\right] \Phi_{c}-i g_{c} C_{\mu}^{\prime} e^{i \gamma_{c}} \Phi_{\operatorname{Im}}+i\left(D_{\mu}^{c^{\prime}} \eta\right) \Phi_{c}}{\Phi_{c}}
\end{aligned}
$$

\footnotetext{
${ }^{6}$ See also a related discussion but with a more explicit calculation on the gauge covariance of field strength in Ref. [4]
} 


$$
\begin{aligned}
& =\frac{\left[\partial_{\mu}-i g\left(A_{j}+\frac{1}{g} D_{j}^{c^{\prime}} \eta\right)\right] \Phi_{c}-i g_{c}\left(C_{j}+\frac{1}{g_{c}} \partial_{j} \gamma_{c}\right) e^{i \gamma_{c}} \Phi_{\operatorname{Im}}+i\left(D_{\mu}^{c^{\prime}} \eta\right) \Phi_{c}}{\Phi_{c}} \\
& = \begin{cases}\frac{D_{\mu}^{A, c, I m} \Phi}{\Phi} & \text { if } \gamma_{c} \in \pi \mathbb{Z}_{\text {even }}, \\
\frac{D_{\mu}^{A, c, I m}\left(\Phi^{\dagger}\right)}{\Phi^{\dagger}} & \text { if } \gamma_{c} \in \pi \mathbb{Z}_{\text {odd }} .\end{cases}
\end{aligned}
$$

Here some of the equalities hold when we focus on the leading order contribution for the gauge transformations. Note that $\partial_{\mu} \Phi_{c}$ can contribute a $\partial_{\mu}\left(e^{i \gamma_{c}} \Phi_{\operatorname{Im}}\right)=i\left(\partial_{\mu} \gamma_{c}\right) e^{i \gamma_{c}} \Phi_{\operatorname{Im}}+\cdots$ that cancels with $-i g_{c}\left(\frac{1}{g_{c}} \partial_{j} \gamma_{c}\right) e^{i \gamma_{c}} \Phi_{\operatorname{Im}}$. We can define a complex conjugation operator of $D_{\mu}^{A, c, \operatorname{Im}}$ as

$$
D_{\mu}^{\dagger A, c, \operatorname{Im}}:=\left(D_{\mu}^{A, c, \operatorname{Im}}\right)^{\dagger} .
$$

Similarly, we find under the gauge transformation $\Phi \rightarrow$ $e^{i \eta(x)} \Phi_{c}$

$$
\begin{aligned}
& D_{\mu}^{\dagger A, c, \operatorname{Im}} \log \Phi^{\dagger} \rightarrow \begin{cases}\frac{D_{\mu}^{D_{A}, c, \mathrm{Im}} \Phi^{\dagger}}{\Phi^{\dagger}} & \text { if } \gamma_{c} \in \pi \mathbb{Z}_{\mathrm{even}}, \\
\frac{D_{\mu, A, c, \mathrm{Im}}(\Phi)}{\Phi} & \text { if } \gamma_{c} \in \pi \mathbb{Z}_{\mathrm{odd}},\end{cases} \\
& D_{\mu}^{A, c, \operatorname{Im}} \log \Phi^{\dagger} \rightarrow \begin{cases}\frac{D_{\mu}^{A, c, I m} \Phi^{\dagger}}{\Phi^{\dagger}} & \text { if } \gamma_{c} \in \pi \mathbb{Z}_{\mathrm{even}}, \\
\frac{D_{\mu}^{A, c, I m}(\Phi)}{\Phi} & \text { if } \gamma_{c} \in \pi \mathbb{Z}_{\mathrm{odd}},\end{cases} \\
& D_{\mu}^{\dagger A, c, \operatorname{Im}} \log \Phi \rightarrow \begin{cases}\frac{D_{\mu}^{\dagger A, c, I \mathrm{Im}} \Phi}{\Phi} & \text { if } \gamma_{c} \in \pi \mathbb{Z}_{\mathrm{even}}, \\
\frac{D_{\mu}^{\dagger A, c, \mathrm{Im}}\left(\Phi^{\dagger}\right)}{\Phi^{\dagger}} & \text { if } \gamma_{c} \in \pi \mathbb{Z}_{\text {odd }} .\end{cases}
\end{aligned}
$$

Similar discussions follow after taking the factor $( \pm)^{\rtimes}$ into the account. Since the denominators in Eqs. (2.41), (2.43), (2.44), and (2.45) are all gauge covariant or complex conjugation gauge covariant:

$$
\Phi \rightarrow e^{( \pm)^{\rtimes} i \eta(x)} \Phi_{c} .
$$

This means that the numerators are also gauge covariant or complex conjugation gauge covariant. We can construct the gauge invariant quantity via pairing the gauge-covariant term with its complex conjugation, and pairing the complex conjugation gauge-covariant term also with its complex conjugation. So we obtain ${ }^{7}$

$$
\begin{aligned}
& \left(D_{\mu}^{A, c, \operatorname{Im}} \Phi\right)\left(D_{A, c, \operatorname{Im}}^{\dagger \mu} \Phi^{\dagger}\right)+\left(D_{\mu}^{\dagger A, c, \operatorname{Im}} \Phi\right)\left(D_{A, c, \operatorname{Im}}^{\mu} \Phi^{\dagger}\right) \\
& =\left(\left(\partial_{\mu}-i g A_{\mu}\right) \Phi-i g_{c} C_{\mu} \Phi_{\operatorname{Im}}\right)\left(\left(\partial^{\mu}+i g A^{\mu}\right) \Phi^{\dagger}\right.
\end{aligned}
$$

\footnotetext{
${ }^{7}$ Here we are allowed to flip the sign $C \rightarrow-C$ since $C$ is only a $\mathbb{Z}_{2}$ gauge field. More precisely, $\oint C=\frac{2 \pi}{2} \mathbb{Z}=\pi \mathbb{Z} \bmod 2 \pi$, while $\oint C=-\oint C \bmod 2 \pi$. It may also look peculiar that the particle $\Phi$ and antiparticle $\Phi^{\dagger}$ both couple to the gauge field $A$ with both \pm 1 couplings. However, we may comfort the readers by reminding the fact that the particle-hole conjugation symmetry $\mathbb{Z}_{2}^{C}$ is already gauged, thus the $\mathbb{Z}_{2}^{C}$ gauge field couples to both the particle $\Phi$ and antiparticle $\Phi^{\dagger}$, via the $\Phi_{\text {Im }}$ part. Furthermore, the $\mathbb{Z}_{2}^{C}$ gauge field can flip the sign of its $U(1)$ gauge charge $+1 \leftrightarrow-1$. This seems to suggest that particle and antiparticle may share part of the degree of freedom. This reminds us the famous fact that the Majorana fermion has the particle and antiparticle identified as the same, although we should beware that our particle $\Phi$ is bosonic instead.
}

$$
\begin{aligned}
& \left.+i g_{c} C^{\mu} \Phi_{\operatorname{Im}}\right)+\left(\left(\partial_{\mu}+i g A_{\mu}\right) \Phi+i g_{c} C_{\mu} \Phi_{\operatorname{Im}}\right) \\
& \times\left(\left(\partial^{\mu}-i g A^{\mu}\right) \Phi^{\dagger}-i g_{c} C^{\mu} \Phi_{\operatorname{Im}}\right) .
\end{aligned}
$$

Approach 2: Yang-Mills method. Let us cross check the above result from a more conventional Yang-Mills method [31]. To start with, we observe that $(d+X)\left({ }_{\Phi_{\mathrm{Im}}}^{\Phi_{\mathrm{Im}}}\right)$ must be gauge covariant under the gauge transformation. Because under a generic gauge transformation $V_{\mathrm{O}(2)}$ it demands $(d+$ $X) \rightarrow\left(V_{\mathrm{O}(2)}(d+X) V_{\mathrm{O}(2)}^{-1}\right)$ and $\left(\begin{array}{c}\Phi_{\mathrm{Re}} \\ \Phi_{\mathrm{Im}}\end{array}\right) \rightarrow\left[V_{\mathrm{O}(2)}\left(\begin{array}{c}\Phi_{\mathrm{Re}} \\ \Phi_{\mathrm{Im}}\end{array}\right]\right.$, we show the gauge covariance of

$$
\begin{aligned}
& (d+X)\left(\begin{array}{c}
\Phi_{\mathrm{Re}} \\
\Phi_{\mathrm{Im}}
\end{array}\right) \rightarrow\left(V_{\mathrm{O}(2)}(d+X) V_{\mathrm{O}(2)}^{-1}\right)\left[V_{\mathrm{O}(2)}\left(\begin{array}{c}
\Phi_{\mathrm{Re}} \\
\Phi_{\mathrm{Im}}
\end{array}\right)\right] \\
& =V_{\mathrm{O}(2)}(d+X)\left(\begin{array}{c}
\Phi_{\mathrm{Re}} \\
\Phi_{\mathrm{Im}}
\end{array}\right) .
\end{aligned}
$$

Since all components of $A, C, \Phi_{\mathrm{Re}}, \Phi_{\operatorname{Im}} \in \mathbb{R}$ are reals, we can pair the gauge covariant term with its transpose (the Hodge dual $\star$ ) to obtain a gauge invariant Lagrangian term (again see footnote 7)

$$
\begin{aligned}
(d+ & X)\left(\begin{array}{c}
\Phi_{\mathrm{Re}} \\
\Phi_{\mathrm{Im}}
\end{array}\right) \wedge \star(d+X)\left(\begin{array}{c}
\Phi_{\mathrm{Re}} \\
\Phi_{\mathrm{Im}}
\end{array}\right) \\
= & \left|\left(\begin{array}{cc}
\partial_{\mu} & g A_{\mu} \\
-g A_{\mu} & \partial_{\mu}-g_{c} C_{\mu}
\end{array}\right)\left(\begin{array}{c}
\Phi_{\mathrm{Re}} \\
\Phi_{\mathrm{Im}}
\end{array}\right)\right|^{2} \\
= & \left|\left(\begin{array}{c}
\partial_{\mu} \Phi_{\mathrm{Re}}+g A_{\mu} \Phi_{\mathrm{Im}} \\
\partial_{\mu} \Phi_{\mathrm{Im}}-g A_{\mu} \Phi_{\mathrm{Re}}-g_{c} C_{\mu} \Phi_{\mathrm{Im}}
\end{array}\right)\right|^{2} \\
= & \left(\partial_{\mu} \Phi_{\mathrm{Re}}+g A_{\mu} \Phi_{\mathrm{Im}}\right)^{2}+\left(\partial_{\mu} \Phi_{\mathrm{Im}}-g A_{\mu} \Phi_{\mathrm{Re}}-g_{c} C_{\mu} \Phi_{\mathrm{Im}}\right)^{2} \\
= & \left(\partial_{\mu} \Phi_{\mathrm{Re}}+g A_{\mu} \Phi_{\mathrm{Im}}\right)^{2}+\left(\partial_{\mu} \Phi_{\mathrm{Im}}-g A_{\mu} \Phi_{\mathrm{Re}}\right)^{2} \\
& +\left(g_{c} C_{\mu} \Phi_{\mathrm{Im}}\right)^{2}-2\left(\partial_{\mu} \Phi_{\mathrm{Im}}-g A_{\mu} \Phi_{\mathrm{Re}}\right)\left(g_{c} C^{\mu} \Phi_{\mathrm{Im}}\right) \\
= & D_{\mu}^{A} \Phi D^{\dagger A, \mu} \Phi^{\dagger}+\left(g_{c} C_{\mu} \Phi_{\mathrm{Im}}\right)^{2}-2\left(\partial_{\mu} \Phi_{\mathrm{Im}}-g A_{\mu} \Phi_{\mathrm{Re}}\right) \\
& \times\left(g_{c} C^{\mu} \Phi_{\mathrm{Im}}\right)=\left(D_{\mu}^{A, c, \operatorname{Im}} \Phi\right)\left(D_{A, c, \operatorname{Im}}^{\dagger \mu} \Phi^{\dagger}\right) .
\end{aligned}
$$




$$
\left(D_{\mu}^{A, c, \operatorname{Im}} \Phi\right)\left(D_{A, c, \operatorname{Im}}^{\dagger \mu} \Phi^{\dagger}\right)=(d+X)\left(\begin{array}{c}
\Phi_{\mathrm{Re}} \\
\Phi_{\mathrm{Im}}
\end{array}\right) \wedge \star(d+X)\left(\begin{array}{c}
\Phi_{\mathrm{Re}} \\
\Phi_{\mathrm{Im}}
\end{array}\right) .
$$

Similarly (again see footnote 7),

$$
\left(D_{\mu}^{A, c, \operatorname{Im}} \Phi^{\dagger}\right)\left(D_{A, c, \operatorname{Im}}^{\dagger \mu} \Phi\right)=(d-X)\left(\begin{array}{c}
\Phi_{\mathrm{Re}} \\
\Phi_{\mathrm{Im}}
\end{array}\right) \wedge \star(d-X)\left(\begin{array}{c}
\Phi_{\mathrm{Re}} \\
\Phi_{\mathrm{Im}}
\end{array}\right)=(d+X)\left(\begin{array}{c}
\Phi_{\mathrm{Re}} \\
-\Phi_{\mathrm{Im}}
\end{array}\right) \wedge \star(d+X)\left(\begin{array}{c}
\Phi_{\mathrm{Re}} \\
-\Phi_{\mathrm{Im}}
\end{array}\right) .
$$

Thus we can construct an $\mathrm{O}(2)$ gauged matter field theory contains a Lagrangian term, Eq. (2.47), and a potential $V\left(|\Phi|^{2}\right)$ as

$$
\left(D_{\mu}^{A, c, \operatorname{Im}} \Phi\right)\left(D_{A, c, \operatorname{Im}}^{\dagger \mu} \Phi^{\dagger}\right)+\left(D_{\mu}^{\dagger A, c, \operatorname{Im}} \Phi\right)\left(D_{A, c, \operatorname{Im}}^{\mu} \Phi^{\dagger}\right)+V\left(|\Phi|^{2}\right) \text {. }
$$

The theory contains particle $\Phi$ and antiparticle $\Phi^{\dagger}$ pair together in an intricate way because the particle-hole $\mathbb{Z}_{2}^{C}$ symmetry $\Phi \stackrel{\mathbb{Z}_{2}^{C}}{\longleftrightarrow} \Phi^{\dagger}$ is also dynamically gauged. If the particle $\Phi$ has a gauge charge 1 , then the antiparticle $\Phi^{\dagger}$ has a gauge charge $(-1)$ under the $\mathrm{U}(1)$ gauge group. (However, see also footnote 7.)

Following $[4,6]$, we can consider the $N$-layer generalization of the theories with $\left(\mathbb{Z}_{2}^{C}\right)^{N}$ gauged. Also, by including the $\mathrm{O}(2)$ Yang-Mills kinetic term, Eq. (2.30), and the level-2 BF theory into the $\mathrm{O}(2)$ gauge matter theory, Eq. (2.53), we are allowed to introduce the twisted cocycle $\omega_{d+1} \in \mathrm{H}^{d+1}\left(\left(\mathbb{Z}_{2}^{C}\right)^{N}, \mathbb{R} / \mathbb{Z}\right)$ from a group cohomology datum [21] to specify the interlayer interactions among $N$ layers. We can write down a schematic path integral:

$$
\begin{aligned}
\mathbf{Z}_{\mathrm{rk}-2-\mathrm{NAb}-\Phi}= & \int\left(\prod_{I=1}^{N}\left[\mathcal{D} A_{I}\right]\left[\mathcal{D} B_{I}\right]\left[\mathcal{D} C_{I}\right]\left[\mathcal{D} \Phi_{I}\right]\left[\mathcal{D} \Phi_{I}^{\dagger}\right]\right) \exp \left[i \int _ { M ^ { d + 1 } } d ^ { d + 1 } x \left(\sum _ { I = 1 } ^ { N } \left[\left|\hat{F}_{\mu \nu}^{c, I}\right|^{2}\right.\right.\right. \\
& \left.\left.\left.+\left(D_{\mu}^{A, c, \operatorname{Im}} \Phi_{I}\right)\left(D_{A, c, \operatorname{Im}}^{\dagger \mu} \Phi_{I}^{\dagger}\right)+\left(D_{\mu}^{\dagger A, c, \operatorname{Im}} \Phi_{I}\right)\left(D_{A, c, \operatorname{Im}}^{\mu} \Phi_{I}^{\dagger}\right)+V\left(\left\{\left|\Phi_{I}\right|^{2}\right\}\right)\right]+\frac{2}{2 \pi} B_{I} d C_{I}\right)\right] \cdot \omega_{d+1}\left(\left\{C_{I}\right\}\right) .
\end{aligned}
$$

\section{B. Degree-1 polynomial symmetry to Pretko's field theory and non-Abelian generalization}

\section{Global-covariant 2-derivative}

Now we construct a field theory that preserves a degree1 polynomial symmetry with a polynomial $Q(x)=\left(\Lambda_{k} x_{k}+\right.$ $\left.\Lambda_{0}\right)$. A degree-1 polynomial symmetry transforms $\Phi$ and $\log \Phi$ as

$$
\Phi \rightarrow e^{i Q(x)} \Phi=e^{i\left(\Lambda_{k} x_{k}+\Lambda_{0}\right)} \Phi
$$

$\log \Phi \rightarrow \log \Phi+i Q(x)=\log \Phi+i\left(\Lambda_{i} x_{i}+\Lambda_{0}\right)$.

Taking $\partial_{x_{i}} \partial_{x_{j}}:=\partial_{i} \partial_{j}$ on both sides, we construct a globally invariant term,

$$
\partial_{i} \partial_{j} \log \Phi \rightarrow \partial_{i} \partial_{j} \log \Phi
$$

We also define

$$
\partial_{i} \partial_{j} \log \Phi:=\frac{P_{i, j}\left(\Phi, \partial \Phi, \partial^{2} \Phi\right)}{\Phi^{2}}=\frac{\Phi \partial_{i} \partial_{j} \Phi-\left(\partial_{i} \Phi\right)\left(\partial_{j} \Phi\right)}{\Phi^{2}} .
$$

Under $\Phi \rightarrow e^{i Q(x)} \Phi$, since the denominator $\Phi^{2} \rightarrow$ $e^{i 2 Q(x)} \Phi^{2}$, so does the numerator $P_{i, j}\left(\Phi, \partial \Phi, \partial^{2} \Phi\right) \rightarrow$ $e^{i 2 Q(x)} P_{i, j}\left(\Phi, \partial \Phi, \partial^{2} \Phi\right)$, which we name

$$
P_{i, j}\left(\Phi, \partial \Phi, \partial^{2} \Phi\right):=\Phi \partial_{i} \partial_{j} \Phi-\left(\partial_{i} \Phi\right)\left(\partial_{j} \Phi\right)
$$

as a global-covariant 2-derivative term, in order to maintain the $\partial_{i} \partial_{j} \log \Phi$ invariant. The gauge invariant Lagrangian con- tains

$$
\begin{aligned}
\left|P_{i, j}\right|^{2}+V\left(|\Phi|^{2}\right):= & P_{i, j}(\Phi) P^{i, j}\left(\Phi^{\dagger}\right)+V\left(|\Phi|^{2}\right) \\
= & \left(\Phi \partial_{i} \partial_{j} \Phi-\partial_{i} \Phi \partial_{j} \Phi\right)\left(\Phi^{\dagger} \partial^{i} \partial^{j} \Phi^{\dagger}\right. \\
& \left.-\partial^{i} \Phi \partial^{j} \Phi^{\dagger}\right)+V\left(|\Phi|^{2}\right) .
\end{aligned}
$$

In this way, based on the systematic method of Ref. [6], we can rederive a Lagrangian formulation of Pretko from 2018 [2], which was recently revisited in [3,5] and [4] from other field theory perspectives.

\section{Gauge-covariant 2-derivative}

To gauge a degree-0 polynomial symmetry, we rewrite $Q(x)$ as a local gauge parameter $\eta(x)$,

$$
\begin{aligned}
\Phi & \rightarrow e^{i \eta(x)} \Phi, \\
\partial_{i} \partial_{j} \log \Phi & \rightarrow \partial_{i} \partial_{j} \log \Phi+i \partial_{i} \partial_{j} \eta(x) .
\end{aligned}
$$

Then $\partial_{i} \partial_{j} \log \Phi$ is no longer an invariant term. This implies that we can write a gauge covariant operator $D_{i, j}[\{\Phi\}]$ via combining $P_{i, j}$ and $A_{i, j}$ :

$$
\begin{aligned}
P_{i, j}\left(\Phi, \partial \Phi, \partial^{2} \Phi\right) & :=\left[\Phi \partial_{i} \partial_{j} \Phi-\left(\partial_{i} \Phi\right)\left(\partial_{j} \Phi\right)\right] \\
& \rightarrow e^{i 2 \eta(x)}\left[P_{i, j}\left(\Phi, \partial \Phi, \partial^{2} \Phi\right)+i \partial_{i} \partial_{j} \eta(x)\right], \\
A_{i, j} & \rightarrow A_{i, j}+\frac{1}{g} \partial_{i} \partial_{j} \eta,
\end{aligned}
$$




$$
\begin{aligned}
D_{i, j}^{A}[\{\Phi\}] & :=P_{i, j}\left(\Phi, \partial \Phi, \partial^{2} \Phi\right)-i g A_{i, j} \Phi^{2} \\
& =\left[\Phi \partial_{i} \partial_{j} \Phi-\left(\partial_{i} \Phi\right)\left(\partial_{j} \Phi\right)-i g A_{i, j} \Phi^{2}\right], \\
D_{i, j}^{A}[\{\Phi\}] & \rightarrow e^{i 2 \eta(x)} D_{i, j}^{A}[\{\Phi\}] .
\end{aligned}
$$

We shall call $D_{i, j}^{A}[\{\Phi\}]$ a gauge covariant 2-derivative term. ${ }^{8}$ So a gauge invariant Lagrangian term can be obtained by complex-conjugate pairing the gauge covariant operator as

$$
\left|D_{i, j}^{A}[\{\Phi\}]\right|^{2}+V\left(|\Phi|^{2}\right)=D_{i, j}^{A}[\{\Phi\}] D_{A}^{\dagger i, j}\left[\left\{\Phi^{\dagger}\right\}\right]+V\left(|\Phi|^{2}\right) .
$$

Thus we also reproduce Pretko's Abelian gauge theory [2].

${ }^{8}$ Since $D_{i, j}^{A}[\{\Phi\}] \rightarrow e^{i 2 \eta(x)} D_{i, j}^{A}[\{\Phi\}]$ with a covariant factor of power 2 as $e^{i 2 \eta(x)}$, we may call this a "2-covariant" for convenience.

\section{Gauge-covariant non-Abelian $\left[\mathrm{U}(1)_{x_{(d)}} \rtimes \mathbb{Z}_{2}^{C}\right]$ rank-3 field strength}

Following Refs. [4,6], we define a non-Abelian rank-3 field strength

$$
\begin{aligned}
\hat{F}_{\mu \nu \xi}^{c}:= & D_{\mu}^{c} A_{\nu \xi}-D_{\nu}^{c} A_{\mu \xi}:=\left(\partial_{\mu}-i g_{c} C_{\mu}\right) A_{\nu \xi} \\
& -\left(\partial_{\nu}-i g_{c} C_{\nu}\right) A_{\mu \xi} .
\end{aligned}
$$

Under the gauge transformation

$$
\begin{aligned}
& A_{\mu \nu} \rightarrow e^{i \gamma_{c}(x)} A_{\mu \nu}+( \pm)^{\rtimes} \frac{1}{2 g}\left(D_{\mu}^{c} D_{\nu}^{c}+D_{\nu}^{c} D_{\mu}^{c}\right)\left[\eta_{v}(x)\right] \\
& =\left\{\begin{array}{l}
V_{\mathbb{Z}_{2}} V_{\mathrm{U}(1)} A_{\mu \nu}=e^{i \gamma_{c}(x)} A_{\mu \nu}+\frac{1}{2 g}\left(D_{\mu}^{c} D_{\nu}^{c}+D_{\nu}^{c} D_{\mu}^{c}\right) \eta_{v}, \\
V_{\mathrm{U}(1)} V_{\mathbb{Z}_{2}} A_{\mu \nu}=e^{i \gamma_{c}(x)}\left[A_{\mu \nu}+\frac{1}{2 g}\left(D_{\mu}^{c} D_{\nu}^{c}+D_{\nu}^{c} D_{\mu}^{c}\right) \eta_{\nu}\right],
\end{array}\right. \\
& C_{\nu} \rightarrow C_{\nu}+\frac{1}{g_{c}} \partial_{\nu} \gamma_{c}(x)
\end{aligned}
$$

we can again show $\hat{F}_{\mu \nu \xi}^{c}$ is gauge covariant:

$$
\hat{F}_{\mu \nu \xi}^{c} \rightarrow e^{i \gamma_{c}(x)} \hat{F}_{\mu \nu \xi}^{c}+\frac{1}{2 g}\left(D_{\mu}^{c}\left(D_{\nu}^{c} D_{\xi}^{c}+D_{\xi}^{c} D_{\nu}^{c}\right)-D_{\nu}^{c}\left(D_{\mu}^{c} D_{\xi}^{c}+D_{\xi}^{c} D_{\mu}^{c}\right)\right) \eta_{v}=e^{i \gamma_{c}(x)} \hat{F}_{\mu \nu \xi}^{c} .
$$

This is true because, under the locally flat $d C=0$ condition, we had derived $\left(D_{\mu}^{c} D_{v}^{c}-D_{\nu}^{c} D_{\mu}^{c}\right)=0$ in Eq. (2.32); furthermore

$$
\begin{aligned}
& \left(D_{\mu}^{c}\left(D_{\nu}^{c} D_{\xi}^{c}+D_{\xi}^{c} D_{\nu}^{c}\right)-D_{\nu}^{c}\left(D_{\mu}^{c} D_{\xi}^{c}+D_{\xi}^{c} D_{\mu}^{c}\right)\right) \\
& =\left(D_{\mu}^{c} D_{v}^{c}-D_{v}^{c} D_{\mu}^{c}\right) D_{\xi}^{c}+\left(D_{\mu}^{c} D_{\xi}^{c} D_{v}^{c}-D_{v}^{c} D_{\xi}^{c} D_{\mu}^{c}\right) \\
& =\left(D_{\mu}^{c} D_{\xi}^{c} D_{v}^{c}-D_{\nu}^{c} D_{\xi}^{c} D_{\mu}^{c}\right)=\left(\partial_{\mu}-i g_{c} C_{\mu}\right)\left(\partial_{\xi}-i g_{c} C_{\xi}\right)\left(\partial_{\nu}-i g_{c} C_{\nu}\right)-\left(\partial_{\nu}-i g_{c} C_{v}\right)\left(\partial_{\xi}-i g_{c} C_{\xi}\right)\left(\partial_{\mu}-i g_{c} C_{\mu}\right) \\
& =\left(\partial_{\mu}-i g_{c} C_{\mu}\right)\left(\partial_{\xi} \partial_{\nu}-i g_{c} C_{\xi} \partial_{\nu}-i g_{c}\left(\partial_{\xi} C_{\nu}\right)-i g_{c} C_{\nu} \partial_{\xi}-g_{c}^{2} C_{\xi} C_{v}\right)-(\mu \leftrightarrow v) \\
& =-i g_{c}\left(\partial_{\xi} C_{v}\right)\left(\partial_{\mu}-i g_{c} C_{\mu}\right)-i g_{c}\left(\partial_{\mu} C_{\xi}\right)\left(\partial_{v}-i g_{c} C_{v}\right)-(\mu \leftrightarrow v) \\
& =\left.i g_{c}\left[(d C)_{\nu \xi}\left(\partial_{\mu}-i g_{c} C_{\mu}\right)-(d C)_{\xi \mu}\left(\partial_{\nu}-i g_{c} C_{\nu}\right)\right]\right|_{d C=0}=0 .
\end{aligned}
$$

The $(\mu \leftrightarrow v)$ are the terms exchanging $\mu$ and $v$ with respect to the previous term. The non-Abelian field strength first appeared in Refs. [4,6]. The gauge-invariant non-Abelian gauge field kinetic Lagrangian term corresponds to

$$
\left|\hat{F}_{\mu \nu \xi}^{c}\right|^{2}:=\hat{F}_{\mu \nu \xi}^{c} \hat{F}^{\dagger c \mu \nu \xi} .
$$

\section{Non-Abelian $\left[\mathrm{U}(1)_{x_{(d)}} \rtimes \mathbb{Z}_{2}^{C}\right]$ gauged matter: Polynomial invariant method}

Following the first approach in Sec. II A 4, we construct non-Abelian gauged matter theory. By generalizing Eq. (2.58), we consider

$$
\begin{aligned}
D_{\mu}^{c, \operatorname{Im}} D_{v}^{c, \operatorname{Im}} \log \Phi= & D_{\mu}^{c, \operatorname{Im}}\left[\left(D_{v}^{c, \operatorname{Im}} \Phi\right) \Phi^{-1}\right]=D_{\mu}^{c, \operatorname{Im}}\left(\left(D_{v}^{c, \operatorname{Im}} \Phi\right) \frac{\Phi^{\dagger}}{|\Phi|^{2}}\right) \\
& =\left(\frac{\Phi\left(D_{\mu}^{c, \operatorname{Im}} D_{v}^{c, \operatorname{Im}} \Phi\right)}{\Phi^{2}}\right)+\left(D_{v}^{c, \operatorname{Im}} \Phi\right)\left(D_{\mu}^{c, \operatorname{Im}} \Phi^{-1}\right) \\
& =\left(\frac{\Phi\left(D_{\mu}^{c, \operatorname{Im}} D_{v}^{c, \operatorname{Im}} \Phi\right)}{\Phi^{2}}\right)+\left(D_{v}^{c, \operatorname{Im}} \Phi\right)\left(D_{\mu}^{c, \operatorname{Im}} \frac{\Phi_{\mathrm{Re}}-i \Phi_{\operatorname{Im}}}{\Phi_{\mathrm{Re}}^{2}+\Phi_{\operatorname{Im}}^{2}}\right) \\
& =\left(\frac{\Phi\left(D_{\mu}^{c, \operatorname{Im}} D_{v}^{c, \operatorname{Im}} \Phi\right)}{\Phi^{2}}\right)+\frac{-\left(D_{\mu}^{c, \operatorname{Im}} \Phi\right)\left(D_{v}^{c, \operatorname{Im}} \Phi\right)+g_{c} C_{\mu}\left(\frac{-2 i \Phi_{\operatorname{mim}}^{2} \Phi}{|\Phi|^{2}}\right)\left(D_{v}^{c, \operatorname{Im}} \Phi\right)}{\Phi^{2}} .
\end{aligned}
$$

In Sec. II B 2, we had learned that for the Abelian gauge sector we require the introduction of a symmetric tensor gauge field $A_{\mu, v}$ in order to cancel the gauge transformation $\partial_{i} \partial_{j} \eta$ between Eq. (2.63) and Eq. (2.64). Thus, we also symmetrize the above 
equation ${ }^{9}$ in order to naturally couple to a symmetric tensor gauge field later:

$$
\frac{\left\{D_{\mu}^{c, \operatorname{Im}}, D_{v}^{c, \operatorname{Im}}\right\}_{+}}{2} \log \Phi=\frac{\left[\Phi \frac{\left\{D_{\mu}^{c, \operatorname{Im}}, D_{v}^{c, \operatorname{Im}}\right\}_{+}}{2} \Phi-D_{\mu}^{c, \operatorname{Im}} \Phi D_{v}^{c, \operatorname{Im}} \Phi+g_{c}\left(\frac{-i \Phi_{\operatorname{Im}}^{2}}{\Phi^{\grave{i}}}\right)\left[\left(C_{\mu} D_{v}^{c, \operatorname{Im}}+C_{\nu} D_{\mu}^{c, \operatorname{Im}}\right) \Phi\right]\right]}{\Phi^{2}} .
$$

The $\left[\mathrm{U}(1)_{x_{(d)}} \rtimes \mathbb{Z}_{2}^{C}\right]$ gauged transformations are

$$
\begin{aligned}
A_{\mu \nu} & \rightarrow A_{\mu \nu}^{\prime}=e^{i \gamma_{c}(x)} A_{\mu \nu}+( \pm)^{\rtimes} \frac{1}{2 g}\left(D_{\mu}^{c} D_{\nu}^{c}+D_{\nu}^{c} D_{\mu}^{c}\right)\left[\eta_{v}(x)\right]=e^{i \gamma_{c}(x)} A_{\mu \nu}+( \pm)^{\rtimes} \frac{1}{2 g}\left[\left\{D_{\mu}^{c}, D_{\nu}^{c}\right\}_{+} \eta_{v}(x)\right], \\
C_{j} & \rightarrow C_{j}^{\prime}=C_{j}+\frac{1}{g_{c}} \partial_{j} \gamma_{c}(x), \\
\Phi & \rightarrow e^{( \pm)^{\rtimes} i \eta(x)} \Phi_{c}=e^{( \pm)^{\rtimes} i \eta(x)}\left(\Phi_{\mathrm{Re}}+i e^{i \gamma_{c}(x)} \Phi_{\operatorname{Im}}\right)= \begin{cases}e^{i \eta(x)} \Phi & \text { if } \gamma_{c} \in \pi \mathbb{Z}_{\text {even }}, \\
e^{( \pm)^{\rtimes} i \eta(x)} \Phi^{\dagger} & \text { if } \gamma_{c} \in \pi \mathbb{Z}_{\text {odd }} .\end{cases}
\end{aligned}
$$

Under the $\left[\mathrm{U}(1)_{x_{(d)}} \rtimes \mathbb{Z}_{2}^{C}\right]$ gauged transformation, $\frac{\left\{D_{\mu}^{c, I m}, D_{v}^{c, I m}\right\}_{+}}{2} \log \Phi$ is not gauge invariant, thus the numerator in Eq. (2.74), $\left[\Phi \frac{\left\{D_{\mu}^{c, \operatorname{Im}}, D_{v}^{c, I m}\right\}_{+}}{2} \Phi-D_{\mu}^{c, \operatorname{Im}} \Phi D_{\nu}^{c, \operatorname{Im}} \Phi+g_{c}\left(\frac{-i \Phi_{\operatorname{Im}}^{2}}{\Phi^{\dagger}}\right)\left[\left(C_{\mu} D_{v}^{c, \operatorname{Im}}+C_{\nu} D_{\mu}^{c, \text { Im }}\right) \Phi\right]\right]$, is also not gauge covariant. This result is what we should expect, because there is a variant term $i \frac{\left\{D_{\mu}^{c, \mathrm{Im}}, D_{v}^{c, \mathrm{Im}}\right\}_{+}}{2} \eta$ in this non-Abelian theory, similar to the variant term $i \partial_{i} \partial_{j} \eta(x)$ that appears in the Abelian version of Eq. (2.63). However, the symmetric tensor gauge field can cancel such a gauge variant term exactly.

So we define a non-Abelian gauge covariant 2-derivative for this non-Abelian theory (generalizing the Abelian case in Sec. II B 2):

$$
D_{\mu, \nu}^{A, c, \operatorname{Im}}[\{\Phi\}]:=\left[\Phi \frac{\left\{D_{\mu}^{c, \mathrm{Im}}, D_{\nu}^{c, \mathrm{Im}}\right\}_{+}}{2} \Phi-D_{\mu}^{c, \operatorname{Im}} \Phi D_{\nu}^{c, \operatorname{Im}} \Phi+g_{c}\left(\frac{-i \Phi_{\operatorname{Im}}^{2}}{\Phi^{\dagger}}\right)\left[\left(C_{\mu} D_{\nu}^{c, \operatorname{Im}}+C_{\nu} D_{\mu}^{c, \operatorname{Im}}\right) \Phi\right]-i g A_{\mu \nu} \Phi^{2}\right] .
$$

Similarly, we have

$$
\begin{aligned}
& D_{\mu, \nu}^{\dagger A, c, \operatorname{Im}}\left[\left\{\Phi^{\dagger}\right\}\right]:=\left[\Phi^{\dagger} \frac{\left\{D_{\mu}^{c, \operatorname{Im} \dagger}, D_{\nu}^{c, \operatorname{Im} \dagger}\right\}_{+}}{2} \Phi^{\dagger}-D_{\mu}^{c, \operatorname{Im} \dagger} \Phi^{\dagger} D_{\nu}^{c, \operatorname{Im} \dagger} \Phi^{\dagger}+g_{c}\left(\frac{+i \Phi_{\operatorname{Im}}^{2}}{\Phi}\right)\left[\left(C_{\mu} D_{\nu}^{c, \operatorname{Im} \dagger}+C_{\nu} D_{\mu}^{c, \operatorname{Im} \dagger}\right) \Phi^{\dagger}\right]+i g A_{\mu \nu} \Phi^{\dagger 2}\right], \\
& D_{\mu, \nu}^{\dagger A, c, \operatorname{Im}}[\{\Phi\}]:=\left[\Phi \frac{\left\{D_{\mu}^{c, \operatorname{Im} \dagger}, D_{\nu}^{c, \operatorname{Im} \dagger}\right\}_{+}}{2} \Phi-D_{\mu}^{c, \operatorname{Im} \dagger} \Phi D_{\nu}^{c, \operatorname{Im} \dagger} \Phi+g_{c}\left(\frac{+i \Phi_{\operatorname{Im}}^{2}}{\Phi^{\dagger}}\right)\left[\left(C_{\mu} D_{\nu}^{c, \operatorname{Im} \dagger}+C_{\nu} D_{\mu}^{c, \operatorname{Im} \dagger}\right) \Phi\right]-i g A_{\mu \nu} \Phi^{2}\right], \\
& D_{\mu, \nu}^{A, c, \operatorname{Im}}\left[\left\{\Phi^{\dagger}\right\}\right]:=\left[\Phi^{\dagger} \frac{\left\{D_{\mu}^{c, \operatorname{Im}}, D_{\nu}^{c, \operatorname{Im}}\right\}_{+}}{2} \Phi^{\dagger}-D_{\mu}^{c, \operatorname{Im}} \Phi^{\dagger} D_{\nu}^{c, \operatorname{Im}} \Phi^{\dagger}+g_{c}\left(\frac{-i \Phi_{\operatorname{Im}}^{2}}{\Phi}\right)\left[\left(C_{\mu} D_{\nu}^{c, \operatorname{Im}}+C_{\nu} D_{\mu}^{c, \operatorname{Im}}\right) \Phi^{\dagger}\right]+i g A_{\mu \nu} \Phi^{\dagger 2}\right] .
\end{aligned}
$$

Therefore Eqs. (2.78) and (2.81) are gauge 2-covariant (see footnote 8) or gauge 2-covariant to their complex conjugate field $\Phi^{\dagger}$ under the gauge transformations (2.75), (2.76), and (2.77):

$$
\begin{aligned}
& D_{\mu, \nu}^{A, c, \operatorname{Im}}[\{\Phi\}] \rightarrow \begin{cases}e^{i 2 \eta(x)} D_{\mu, \nu}^{A, c, \operatorname{Im}}[\{\Phi\}] & \text { if } \gamma_{c} \in \pi \mathbb{Z}_{\mathrm{even}}, \\
e^{i( \pm)^{\rtimes} 2 \eta(x)} D_{\mu, \nu}^{A, c, \operatorname{Im}}\left[\left\{\Phi^{\dagger}\right\}\right] & \text { if } \gamma_{c} \in \pi \mathbb{Z}_{\mathrm{odd}},\end{cases} \\
& D_{\mu, \nu}^{\dagger A, \operatorname{Im}}\left[\left\{\Phi^{\dagger}\right\}\right] \rightarrow \begin{cases}e^{-i 2 \eta(x)} D_{\mu, \nu}^{\dagger A, c, \operatorname{Im}}\left[\left\{\Phi^{\dagger}\right\}\right] & \text { if } \gamma_{c} \in \pi \mathbb{Z}_{\mathrm{even}}, \\
e^{-i( \pm)^{\rtimes} 2 \eta(x)} D_{\mu, \nu}^{\dagger A, c, \operatorname{Im}}[\{\Phi\}] & \text { if } \gamma_{c} \in \pi \mathbb{Z}_{\mathrm{odd}},\end{cases} \\
& D_{\mu, v}^{\dagger A, c, \operatorname{Im}}[\{\Phi\}] \rightarrow \begin{cases}e^{i 2 \eta(x)} D_{\mu, v}^{\dagger A, c, \operatorname{Im}}[\{\Phi\}] & \text { if } \gamma_{c} \in \pi \mathbb{Z}_{\text {even }}, \\
e^{i( \pm)^{\rtimes} 2 \eta(x)} D_{\mu, v}^{\dagger A, c, \operatorname{Im}}\left[\left\{\Phi^{\dagger}\right\}\right] & \text { if } \gamma_{c} \in \pi \mathbb{Z}_{\text {odd }},\end{cases} \\
& D_{\mu, \nu}^{A, c, \operatorname{Im}}\left[\left\{\Phi^{\dagger}\right\}\right] \rightarrow \begin{cases}e^{-i 2 \eta(x)} D_{\mu, \nu}^{A, c, \operatorname{Im}}\left[\left\{\Phi^{\dagger}\right\}\right] & \text { if } \gamma_{c} \in \pi \mathbb{Z}_{\text {even }}, \\
e^{-i( \pm)^{\rtimes} 2 \eta(x)} D_{\mu, \nu}^{A, c, \operatorname{Im}}[\{\Phi\}] & \text { if } \gamma_{c} \in \pi \mathbb{Z}_{\text {odd }} .\end{cases}
\end{aligned}
$$

Following [4,6], we can consider the $N$-layer generalization of the theories with $\left(\mathbb{Z}_{2}^{C}\right)^{N}$ gauged. Also by including the $\left[\mathrm{U}(1)_{x_{(d)}} \rtimes \mathbb{Z}_{2}^{C}\right]$-gauge kinetic term Eq. (2.71) and the level-2 BF theory into the gauge matter theory, again we are allowed

${ }^{9}$ Below we use the notation $\left\{J_{1}, J_{2}\right\}_{+}:=J_{1} J_{2}+J_{2} J_{1}$ to define the anticommutator, e.g.,

$$
\left\{D_{\mu}^{c, \operatorname{Im}}, D_{v}^{c, \operatorname{Im}}\right\}_{+}:=D_{\mu}^{c, \operatorname{Im}} D_{v}^{c, \operatorname{Im}}+D_{v}^{c, \operatorname{Im}} D_{\mu}^{c, \operatorname{Im}} .
$$


to introduce the twisted cocycle $\omega_{d+1} \in \mathrm{H}^{d+1}\left(\left(\mathbb{Z}_{2}^{C}\right)^{N}, \mathbb{R} / \mathbb{Z}\right)$ from a group cohomology datum [21] to specify the interlayer interactions among $N$ layers. We can write down a schematic path integral (see also footnote 7):

$$
\begin{aligned}
\mathbf{Z}_{\mathrm{rk}-3-\mathrm{NAb}-\Phi}= & \int\left(\prod_{I=1}^{N}\left[\mathcal{D} A_{I}\right]\left[\mathcal{D} B_{I}\right]\left[\mathcal{D} C_{I}\right]\left[\mathcal{D} \Phi_{I}\right]\left[\mathcal{D} \Phi_{I}^{\dagger}\right]\right) \exp \left(i \int _ { M ^ { d + 1 } } \sum _ { I = 1 } ^ { N } \left[d ^ { d + 1 } x \left(\left|\hat{F}_{\mu \nu \xi}^{c, I}\right|^{2}\right.\right.\right. \\
& \left.\left.\left.+D_{\mu, \nu}^{A, c, \operatorname{Im}}\left[\left\{\Phi_{I}\right\}\right] D_{A, c, \operatorname{Im}}^{\dagger \mu, \nu}\left[\left\{\Phi_{I}^{\dagger}\right\}\right]+D_{\mu, \nu}^{\dagger A, c, \operatorname{Im}}\left[\left\{\Phi_{I}\right\}\right] D_{A, c, \operatorname{Im}}^{\mu, \nu}\left[\left\{\Phi_{I}^{\dagger}\right\}\right]+V\left(\left\{\left|\Phi_{I}\right|^{2}\right\}\right)\right)+\frac{2}{2 \pi} B_{I} d C_{I}\right]\right) \cdot \omega_{d+1}\left(\left\{C_{I}\right\}\right) .
\end{aligned}
$$

The ingredient in our present work beyond the previous Refs. $[4,6]$ is that now the matter fields directly interact with non-Abelian gauge fields.

\section{Degree-(m-1) polynomial symmetry to non-Abelian higher-rank tensor gauged matter theory}

In this subsection, we outline a generalization of previous Secs. II A and II B to a general degree- $(m-1)$ polynomial symmetry and by gauging it and the particle-hole $\mathbb{Z}_{2}^{C}$ symmetry to obtain a non-Abelian higher-rank tensor gauged matter field theory.

\section{Global symmetry: $\prod_{M=1}^{m-1} \mathrm{U}(1)_{x_{\left(\begin{array}{c}n \\ M\end{array}\right)}^{M}}$}

Follow Ref. [6], a degree- $(m-1)$-polynomial symmetry acts on the complex scalar $\Phi(x) \in C$ :

$$
\Phi \rightarrow e^{i Q(x)} \Phi=e^{i\left(\Lambda_{i_{1}, \ldots, i_{m-1}} x_{i_{1}} \ldots x_{i_{m-1}}+\cdots+\Lambda_{i, j} x_{i} x_{j}+\Lambda_{i} x_{i}+\Lambda_{0}\right)} \Phi .
$$

Different $\Lambda_{\ldots}$ introduce degree U(1) degrees of freedom. Different $\mathrm{U}(1)$ symmetries for different degrees and different $\Lambda_{\text {... commute. We denote such a degree- }(m-1) \text { polynomial }}$ symmetry structure with several U(1) symmetry groups as ${ }^{10}$

$$
\mathrm{U}(1)_{\substack{x_{(m-1}^{m-1} \\
n}} \times \cdots \times \mathrm{U}(1)_{x_{(n)}} \times \mathrm{U}(1)=\prod_{M=1}^{m-1} \mathrm{U}(1)_{x_{\left(\begin{array}{l}
n \\
M
\end{array}\right)}^{M}}
$$

The subindices of $\mathrm{U}(1)$ specifies which $\Lambda \ldots$ degree of freedom contributes such a $\mathrm{U}(1)$. Note that $0 \leqslant M \leqslant m-1$. For example, for the degree-1 polynomial symmetry with different $\Lambda_{j}$, we denote

$$
\mathrm{U}(1)_{x_{(n)}}:=\prod_{j=1}^{n} \mathrm{U}(1)_{x_{j}},
$$

each for different $\Lambda_{j}$. In general, we denote

$$
\mathrm{U}(1)_{x_{\left(\begin{array}{l}
n \\
(M)
\end{array}\right)}^{M}}:=\prod_{\left\{j_{1}, \ldots, j_{M}\right\}} \mathrm{U}(1)_{x_{j_{1}}, \ldots, x_{j_{M}}},
$$

\footnotetext{
${ }^{10}$ Each of the U(1) factors represents a U(1) group. However, the products of these $U(1)$ act differently: the left-most $U(1)$ acts globally as 0 -form symmetry, the second-left-most $\mathrm{U}(1)_{x_{(n)}}$ acts a vector global symmetry, etc. Thus we call it a global symmetry structure (instead of a global symmetry group), because each U(1) has different physical meanings associated with space(time) coordinates. Furthermore, we also refer to its gauging as a gauged structure (not necessarily the same as the conventional gauge group).
}

each for different $\Lambda_{j_{1}, \ldots, j_{M}}$.

\section{2. $\mathbb{Z}_{2}^{C}$ charge-conjugation (particle-hole) symmetry}

In addition to the polynomial symmetry in Sec. II C 1, as noticed in $[4,6]$, we have a $\mathbb{Z}_{2}^{C}$ charge-conjugation (particlehole) symmetry. It acts on the complex scalar $\Phi$ switching from a particle to an antiparticle. The $\mathbb{Z}_{2}^{C}$ symmetry persists even after we gauge the Abelian polynomial-symmetry, which also acts on the rank- $m$ Abelian symmetric tensor $A_{i_{1}, \ldots, i_{m}}$ and the gauge parameter $\eta_{v}(x)$ for $\Phi \rightarrow e^{i \eta_{v}} \Phi$ :

$$
\begin{aligned}
\Phi & \mapsto \Phi^{\dagger}, \\
A_{i_{1}, \ldots, i_{m}} & \mapsto-A_{i_{1}, \ldots, i_{m}}, \\
\eta_{v}(x) & \mapsto-\eta_{v}(x) .
\end{aligned}
$$

The U(1) degree- $(m-1)$ polynomial symmetry does not commute with $\mathbb{Z}_{2}^{C}$ symmetry. Abbreviating Eq. (2.86)'s $\prod_{M=1}^{m-1} \mathrm{U}(1)_{x_{\left(\begin{array}{c}n \\ (n)\end{array}\right)}}$ as $\mathrm{U}(1)_{\text {poly }}$ symmetry,

$$
\begin{aligned}
& U_{\mathbb{Z}_{2}^{c}} U_{\mathrm{U}(1)_{\text {poly }}} \Phi=U_{\mathbb{Z}_{2}^{c}}\left(e^{i Q(x)} \Phi\right)=e^{i Q(x)} \Phi^{\dagger}, \\
& U_{\mathrm{U}(1)_{\text {poly }}} U_{\mathbb{Z}_{2}^{c}} \Phi=U_{\mathrm{U}(1)_{\text {poly }}}\left(\Phi^{\dagger}\right)=e^{-i Q(x)} \Phi^{\dagger} .
\end{aligned}
$$

So we have indeed a non-Abelian/noncommutative global symmetry structure:

$$
\left(\prod_{M=1}^{m} \mathrm{U}(1)_{x_{\left(\begin{array}{c}
n \\
M
\end{array}\right)}^{M}}\right) \rtimes \mathbb{Z}_{2}^{C}:=U_{\mathrm{U}(1)_{\text {poly }}} \rtimes \mathbb{Z}_{2}^{C} .
$$

\section{Non-Abelian/noncommutative gauge structure:

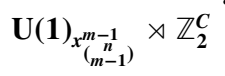

Even after we gauge the $\mathrm{U}(1)$ polynomial symmetry, we can still observe that the gauge transformation of $\left[U_{\mathrm{U}(1) \text { poly }}\right]$ does not commute with the $\mathbb{Z}_{2}^{C}$ global symmetry transformation, which both can act on the $\Phi$ and the rank- $m$ symmetric tensor $A$ respectively:

$$
\begin{aligned}
U_{\mathbb{Z}_{2}^{c}}\left[U_{\mathrm{U}(1)_{\text {poly }}}\right] \Phi & =U_{\mathbb{Z}_{2}^{c}}\left(e^{i \eta_{v}} \Phi\right)=e^{i \eta_{v}} \Phi^{\dagger}, \\
t\left[U_{\mathrm{U}(1)_{\text {poly }}}\right] U_{\mathbb{Z}_{2}^{c}} \Phi & =U_{\mathbb{Z}_{2}^{c}}\left(\Phi^{\dagger}\right)=e^{-i \eta_{v}} \Phi^{\dagger}, \\
U_{\mathbb{Z}_{2}^{c}}\left[U_{\mathrm{U}(1)_{\text {poly }}}\right] A_{i_{1}, \ldots, i_{m}} & =U_{\mathbb{Z}_{2}^{c}}\left(A_{i_{1}, \ldots, i_{m}}+\frac{1}{g} \partial_{\mu} \partial_{\nu} \eta_{v}\right) \\
& =-A_{i_{1}, \ldots, i_{m}}+\frac{1}{g} \partial_{i_{1}} \partial_{i_{2}} \cdots \partial_{i_{m-1}} \partial_{i_{m}} \eta_{v}, \\
{\left[U_{\mathrm{U}(1)_{\text {poly }}}\right] U_{\mathbb{Z}_{2}^{c}} A_{i_{1}, \ldots, i_{m}} } & =U_{\mathbb{Z}_{2}^{c}}\left(-A_{i_{1}, \ldots, i_{m}}\right) \\
& =-A_{i_{1}, \ldots, i_{m}}-\frac{1}{g} \partial_{i_{1}} \partial_{i_{2}} \cdots \partial_{i_{m-1}} \partial_{i_{m}} \eta_{v} .
\end{aligned}
$$


By gauging the degree- $(m-1)$ polynomial symmetry and keeping only the rank- $m$ symmetric tensor $A_{i_{1}, \ldots, i_{m}}$, we are left with a non-Abelian/noncommutative gauge structure:

$$
\mathrm{U}(1)_{\substack{\left(\begin{array}{c}
n \\
(m-1
\end{array}\right) \\
m-1}} \rtimes \mathbb{Z}_{2}^{C} .
$$

\section{Non-Abelian $\left[\mathrm{U}(1)_{\substack{(m-1 \\(m-1)}} \rtimes \mathbb{Z}_{2}^{C}\right]$ gauged matter: Polynomial invariant method}

We propose a polynomial invariant method to generalize the procedure of Sec. II B 4 from a degree-1 polynomial to a generic degree [here a degree- $(m-1)$ polynomial]. First, we determine

$$
D_{i_{1}}^{c, \operatorname{Im}} D_{i_{2}}^{c, \operatorname{Im}} \cdots D_{i_{m}}^{c, \operatorname{Im}} \log \Phi:=\frac{P_{i_{1}, \ldots, i_{m}}^{c}\left(\Phi, \ldots,\left(D^{c, \operatorname{Im}}\right)^{m} \Phi\right)}{\Phi^{m}} .
$$

For example, the degree-1 case is obtained in Eq. (2.72). The degree-2 case is obtained in Ref. [6]. ${ }^{11}$ The functional $P^{c}$ should be a generalization of the result obtained in Ref. [6]. The derivative $\left(D^{c, I m}\right)$ involves the coupling to a 1 -form $C$ gauge field. Moreover, when we turn off the $C$ gauge field, we reduce $D_{i_{1}}^{c, \operatorname{Im}} D_{i_{2}}^{c, \operatorname{Im}} \cdots D_{i_{m}}^{c, \operatorname{Im}} \log \Phi$ to a previous formula obtained in Ref. [6]:

$$
\partial_{i_{1}} \ldots \partial_{i_{m}} \log \Phi:=\frac{P_{i_{1}, \ldots, i_{m}}\left(\Phi, \ldots, \partial^{m} \Phi\right)}{\Phi^{m}} .
$$

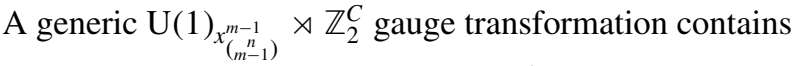

$$
\begin{aligned}
A_{\mu \nu} \rightarrow & A_{\mu \nu}^{\prime}=e^{i \gamma_{c}(x)} A_{\mu \nu}+( \pm)^{\rtimes} \frac{1}{(m !) g}\left(D_{\left(i_{1}\right.}^{c} D_{i_{2}}^{c} \cdots D_{\left.i_{m}\right)}^{c}\right) \\
& \times\left[\eta_{v}(x)\right], \\
C_{j} \rightarrow & C_{j}^{\prime}=C_{j}+\frac{1}{g_{c}} \partial_{j} \gamma_{c}(x), \\
\Phi \rightarrow & e^{( \pm)^{\rtimes} i \eta(x)} \Phi_{c}=e^{( \pm)^{\rtimes} i \eta(x)}\left(\Phi_{\mathrm{Re}}+i e^{i \gamma_{c}(x)} \Phi_{\mathrm{Im}}\right) \\
= & \begin{cases}e^{i \eta(x)} \Phi & \text { if } \gamma_{c} \in \pi \mathbb{Z}_{\mathrm{even}}, \\
e^{( \pm)^{\rtimes} i \eta(x)} \Phi^{\dagger} & \text { if } \gamma_{c} \in \pi \mathbb{Z}_{\mathrm{odd}} .\end{cases}
\end{aligned}
$$

\footnotetext{
${ }^{11}$ For a degree-2 polynomial symmetry, $\Phi \rightarrow e^{i Q(x)} \Phi=$ $e^{i\left(\Lambda_{i, j} x_{i} x_{j}+\Lambda_{i} x_{i}+\Lambda_{0}\right)} \Phi$, we construct a covariant 3-derivative (triple-derivative) below. First, $\log \Phi \rightarrow \log \Phi+i Q(x)=$ $\log \Phi+i\left(\Lambda_{i, j} x_{i} x_{j}+\Lambda_{i} x_{i}+\Lambda_{0}\right)$. We take $\partial_{x_{i}} \partial_{x_{j}} \partial_{x_{k}}:=\partial_{i} \partial_{j} \partial_{k}$ on both sides, $\partial_{i} \partial_{j} \partial_{k} \log \Phi=\frac{P_{i, j, k}\left(\Phi, \ldots, \partial^{3} \Phi\right)}{\Phi^{3}} \rightarrow \partial_{i} \partial_{j} \partial_{k} \log \Phi$, which is globally invariant under the degree-2 polynomial symmetry:

$$
=\frac{\left.\Phi^{2}\left(\partial_{i} \partial_{j} \partial_{k} \Phi\right)-3 \Phi\left(\partial_{(k} \Phi \partial_{i} \partial_{j}\right) \Phi\right)+2\left(\partial_{i} \Phi\right)\left(\partial_{j} \Phi\right)\left(\partial_{k} \Phi\right)}{\Phi^{3}} .
$$$$
\frac{P_{i, j, k}\left(\Phi, \ldots, \partial^{3} \Phi\right)}{\Phi^{3}}
$$

We use the symmetrized tensor notation: $T_{\left(i_{1} i_{2} \cdots i_{k}\right)}=$ $\frac{1}{k !} \sum_{\sigma \in \mathrm{S}_{\mathrm{k}}} T_{i_{\sigma 1} i_{\sigma 2} \cdots i_{\sigma k}}$, with parentheses $(i j k)$ around the indices being symmetrized. The $S_{\mathrm{k}}$ is the symmetric group of $k$ elements. Since the denominator $\Phi^{3} \rightarrow e^{i 3 Q(x)} \Phi^{3}$, so does the numerator $P_{i, j, k}\left(\Phi, \ldots, \partial^{3} \Phi\right) \rightarrow e^{i 3 Q(x)} P_{i, j, k}\left(\Phi, \ldots, \partial^{3} \Phi\right)$, whereby we call the denominator and numerator 3-covariant. The Lagrangian thus contains $\left|P_{i, j, k}\right|^{2}:=P_{i, j, k}(\Phi) P^{i, j, k}\left(\Phi^{\dagger}\right)[6]$.
}

Here $\left(D_{\left(i_{1}\right.}^{c} D_{i_{2}}^{c} \cdots D_{\left.i_{m}\right)}^{c}\right):=\left(D_{i_{1}}^{c} D_{i_{2}}^{c} \cdots D_{i_{m}}^{c}+D_{i_{2}}^{c} D_{i_{1}}^{c} \cdots D_{i_{m}}^{c}+\cdots\right)$ yields a symmetrization over the subindices under the lower bracket $\left(i_{1}, \ldots, i_{m}\right)$, the permutation $(m !)$ terms. The $P_{i_{1}, \ldots, i_{m}}^{c}\left(\Phi, \ldots,\left(D^{c, \mathrm{Im}}\right)^{m} \Phi\right)$ is not gauge covariant under the generic gauge transformation. But we can append the $A$ gauge field to make it gauge covariant:

$$
D_{i_{1}, \ldots, i_{m}}^{A, c, \operatorname{Im}}[\{\Phi\}]:=P_{i_{1}, \ldots, i_{m}}^{c}\left(\Phi, \ldots,\left(D^{c, \operatorname{Im}}\right)^{m} \Phi\right)-i g A_{i_{1}, \ldots, i_{m}} \Phi^{m}
$$

where we implicitly sum over all possible indices as $\sum_{\left\{i_{1}, \ldots, i_{m}\right\}}$ over both the left- and right-hand sides. The special case when $m=1$ is given in Eq. (3.14) and $m=2$ is given in Eq. (2.78). The non-Abelian gauge covariant rank- $(m+1)$ field strength is already obtained and defined in Ref. [6]:

$$
\begin{aligned}
\hat{F}_{\mu, \nu, i_{2}, \ldots, i_{m}}^{c} & :=D_{\mu}^{c} A_{\nu, i_{2}, \ldots, i_{m}}-D_{\nu}^{c} A_{\mu, i_{2}, \ldots, i_{m}} \\
& :=\left(\partial_{\mu}-i g_{c} C_{\mu}\right) A_{\nu, i_{2}, \ldots, i_{m}}-\left(\partial_{\nu}-i g_{c} C_{\nu}\right) A_{\mu, i_{2}, \ldots, i_{m}} .
\end{aligned}
$$

We can include the ingredients of non-Abelian gauge theory coupling to the obtained gauged matter sectors (see footnote 7):Ivskip-3pt

$$
\begin{aligned}
\mathbf{Z}_{\mathrm{rk}-(m+1)-\mathrm{NAb}-\Phi}= & \int\left(\prod_{I=1}^{N}\left[\mathcal{D} A_{I}\right]\left[\mathcal{D} B_{I}\right]\left[\mathcal{D} C_{I}\right]\left[\mathcal{D} \Phi_{I}\right]\left[\mathcal{D} \Phi_{I}^{\dagger}\right]\right) \\
& \times \exp \left(i \int _ { M ^ { d + 1 } } \sum _ { I = 1 } ^ { N } \left[d ^ { d + 1 } x \left(\left|\hat{F}_{\mu, \nu, i_{2}, \ldots, i_{m}}^{c, I}\right|^{2}\right.\right.\right. \\
& +D_{i_{1}, \ldots, i_{m}}^{A, c, \operatorname{Im}}\left[\left\{\Phi_{I}\right\}\right] D_{A, c, I m}^{\dagger i_{1}, \ldots, i_{m}}\left[\left\{\Phi_{I}^{\dagger}\right\}\right] \\
& +D_{i_{1}, \ldots, i_{m}}^{\dagger A, c, i_{m}}\left[\left\{\Phi_{I}\right\}\right] D_{A, c, \operatorname{Im}}^{i_{1}, \ldots, i_{m}}\left[\left\{\Phi_{I}^{\dagger}\right\}\right] \\
& \left.\left.\left.+V\left(\left\{\left|\Phi_{I}\right|^{2}\right\}\right)\right)+\frac{2}{2 \pi} B_{I} d C_{I}\right]\right) \cdot \omega_{d+1}\left(\left\{C_{I}\right\}\right),
\end{aligned}
$$

so we derive a non-Abelian gauged matter field theory. The setup and notations are directly generalized from Sec. II B 4.

\section{NEW SIGMA MODELS IN A FAMILY AND TWO TYPES OF VORTICES}

Section II proposes a family of non-Abelian gauged matter field theories. In this section, we study the "dualized" theory: instead of using the matter field degrees of freedom, we try to incorporate the vortex degrees of freedom into the field theory.

To start with, there are at least two types of vortex degrees of freedom that we can identify.

(1) The complex scalar matter field can be written as:

$$
\begin{aligned}
& \Phi(x)=\sqrt{\rho(x)} \exp [i \phi(x)] \in \mathbb{C}, \\
& \rho(x) \in \mathbb{R}_{\geqslant 0}, \\
& \phi(x) \in[0,2 \pi)+2 \pi \mathbb{Z} .
\end{aligned}
$$

So we can use Eq. (2.96) to replace the path integral measure $\int\left[\mathcal{D} \Phi_{I}\right]\left[\mathcal{D} \Phi_{I}^{\dagger}\right]$ with $\int[\mathcal{D} \rho][\mathcal{D} \phi]$ (up to some phase space volume factor) and also substitute $\Phi_{I}=\sqrt{\rho_{I}} \exp \left(i \phi_{I}\right)$ and $\Phi_{I}^{\dagger}=$ $\sqrt{\rho_{I}} \exp \left(-i \phi_{I}\right){ }^{12}$ The $\mathbb{R}_{\geqslant 0}$ takes non-negative real values.

\footnotetext{
${ }^{12}$ We will capture the integer nonsmooth singular part of $\frac{1}{2 \pi} d d \phi=$ $n \in \mathbb{Z}$ via the Cauchy-Riemann relation, the winding number, and topological degree theory in Sec. IV.
} 
When there are $N$ layers, $I=1, \ldots, N$, there are $N$ flavors of vortex fields $\phi_{I}$.

(2) The antisymmetric tensor TQFT sector (the level-2 $\mathrm{BF}$ theory as a $\mathbb{Z}_{2}$ gauge theory twisted by Dijkgraaf-Witten group cohomology topological terms)

$$
\begin{aligned}
& \int \prod_{I=1}^{N}\left[\mathcal{D} B_{I}\right]\left[\mathcal{D} C_{I}\right] \exp \left[i \int_{M^{d+1}}\left(\sum_{I=1}^{N} \frac{2}{2 \pi} B_{I} d C_{I}\right)\right] \\
& \cdot \omega_{d+1}\left(\left\{C_{I}\right\}\right)
\end{aligned}
$$

can also be regarded as the disordered phase of a sigma model given by another scalar field $\theta_{I}$. The derivations of sigma models governing the ordered-disorder phases relevant for twisted Dijkgraaf-Witten type TQFTs ${ }^{13}$ have been studied in $[32,33,35]$; here we will implement the procedure done in

${ }^{13}$ We recall the approach of Refs. [32,33] and its generalization:

(i) The ordered phase of sigma models describes the weak fluctuations around the symmetry-breaking phases.

(ii) The disorder phases of sigma models describe the strong fluctuations around the symmetry-restored phases as continuum formulations of TQFTs, SETs, or SPTs of Dijkgraaf-Witten type. The U(1) spontaneously symmetry breaking phase has a superfluid ground state, which is an ordered phase with respect to $\phi$ with an order parameter

$$
\langle\exp (i \theta)\rangle \neq 0 .
$$

It is well known that if we disorder the U(1) spontaneously symmetry breaking (superfluid) state, we can obtain a disordered phase known as a gapped insulator [27-29]. Our approach is basically along this line of logical thinking, except that we generalize the approach as follows:

(i) Disorder the ordered phase [U(1) symmetry breaking superfluid] to a disordered phase of gapped topological order (e.g., the $\mathbb{Z}_{N}$-gauge theory, where the $\mathbb{Z}_{1}$-gauge theory means a trivial gapped insulator and the $\mathbb{Z}_{2}$-gauge theory means the low energy theory of deconfined $\mathbb{Z}_{2}$-toric code, $\mathbb{Z}_{2}$-spin liquid, or $\mathbb{Z}_{2}$ superconductor). (Beware that Refs. [32,33] only consider the case of a superfluid-insulator transition for $N=1$; here we consider a superfluid-topological-order transition for a generic $N$.)

(ii) Following [32,33], introduce additional topological multikink Berry phase specified by the cocycle of cohomology group

$$
\omega_{d+1}\left(\left\{C_{I}\right\}\right) \simeq \omega_{d+1}\left(\left\{d \theta_{I}\right\}\right)
$$

to the superfluid.

To comprehend our formalism, here we overview this approach using field theory [32]. We start from the superfluid state in a $d$-spacetime dimension described by a bosonic U(1) quantum phase $\theta$ kinetic term and a superfluid compressibility coefficient $\chi$. The partition function $\mathbf{Z}$ is

$$
\mathbf{Z}=\int[\mathcal{D} \theta] \exp \left(i \int d^{d} x \frac{\chi}{2}\left(\partial_{\mu} \theta_{s}+\partial_{\mu} \theta_{v}\right)^{2}\right) .
$$

The $\theta=\theta_{s}+\theta_{v}$, with a smooth piece $\theta_{s}$ and a singular vortex piece $\theta_{v}$ for the bosonic phase $\theta$. We emphasize that the $\theta_{v}$ is essential to capture the vortex core; see Sec. IV. We introduce an auxiliary field $j^{\mu}$ and apply the Hubbard-Stratonovich technique [34],

$$
\mathbf{Z}=\int[\mathcal{D} \theta]\left[\mathcal{D} j^{\mu}\right] \exp \left(i \int d^{d} x \frac{1}{2 \chi}\left(j_{I}^{\mu}\right)^{2}-i j^{\mu}\left(\partial_{\mu} \theta_{s}+\partial_{\mu} \theta_{v}\right)\right) .
$$

[32]. We write

$$
\theta_{I}=\theta_{s, I}+\theta_{v, I},
$$

where $\theta_{s, I}$ describes the smooth $(s)$ part while the $\theta_{v, I}$ describes the singular vortex $(v)$ part. See footnote 13 and [32].

By integrating out the smooth part $\int\left[D \theta_{s}\right]$, we obtain a constraint $\delta\left(\partial_{\mu} j^{\mu}\right)$ on the path integral measure. Naively, in the antisymmetric tensor differential form notation, the constraint seems to be in disguise,

$$
d(\star j)=0 \quad \text { or } \quad 2 \pi \oint d(\star j)=\mathbb{Z},
$$

and the solution in disguise is $j=\frac{1}{2 \pi}(\star d B)$. (Here we choose a normalization convention.) However, we imagine the procedure is that the $N$-fold vortex of superfluid becomes a trivial object (instead of a 1-fold vortex of superfluid) that can be created or annihilated for free from the $\mathbb{Z}_{N}$-gauge theory vacuum. Instead we may impose a revised constraint

$$
2 \pi \oint d(\star j)=N \mathbb{Z} .
$$

Note that the $2 \pi N$ on the right-hand side means that the $N$-fold of $2 \pi$ vortices comes to be identified as a trivial zero vortex (nonvortex). The solution is, with $\star$ the Hodge star,

$$
j=\frac{N}{2 \pi}(\star d B) \text {. }
$$

We can define a generic form $j^{\mu}=\frac{N}{2 \pi(d-2) !} \epsilon^{\mu \mu_{2} \ldots \mu_{d}} \partial_{\mu_{2}} B_{\mu_{3} \ldots \mu_{d}}$, with an anti-symmetric tensor $B$ with a total spacetime dimension $d$ (most conveniently, we may consider $2 \mathrm{~d}$ space or $2+1 \mathrm{~d}$ spacetime in order to implement a winding number in Sec. IV), to satisfy this constraint. To disorder the superfluid, we have to make the $\theta_{v}$-angle strongly fluctuates - namely we should take the $\chi \rightarrow \infty$ limit to achieve large $\left|\delta \theta_{v}\right|^{2} \gg 1$, the disordered limit of superfluid. Plug in Eq. (3.7), the partition function becomes:

$$
\mathbf{Z}=\int\left[\mathcal{D} \theta_{v}\right][\mathcal{D} B] \exp \left(+\int i \frac{N}{2 \pi} B \wedge\left(d^{2} \theta_{v}\right)\right) .
$$

Hereafter we may compensate the dropped \pm -sign by a fieldredefinition. Although naively $d^{2}=0$, due to the singularity core of $\theta_{v}$, the $\left(d^{2} \theta_{v}\right)$ can be nonzero, see Sec. IV, which implies that (at least for the 2-dimensional space mapping to a deformed $S^{1}$-circle as a target space):

$$
\frac{1}{2 \pi} d^{2} \theta_{v}=n \quad \bmod N, \text { thus } n \in \mathbb{Z}_{N} .
$$

Thus, $\left(d^{2} \theta_{v}\right)$ describes the vortex core density and the vortex current, which we denote

$$
\frac{1}{2 \pi} d^{2} \theta_{v}=\star j_{\text {vortex }} .
$$

In addition, the Noether theorem leads to the conservation of the vortex current: the continuity equation

$$
d \star j_{\text {vortex }}=0
$$

implies that

$$
\star j_{\text {vortex }}=d C /(2 \pi)
$$

for some 1-form gauge field $C$. We can thus define the singular part of bosonic phase

$$
d \theta_{v}=C
$$


The exterior derivative of the vortex field should be identified as the 1 -form $C$ gauge field as

$$
d \theta_{v}=C .
$$

In this way, the TQFT sector of the theory (as a disordered phase of some sigma model) can be rewritten as a sigma model with the vortex field $\theta_{v}$ degree of freedom:

$$
\begin{aligned}
& \int\left(\prod_{I=1}^{N}\left[\mathcal{D} B_{I}\right]\left[\mathcal{D} \theta_{s, I}\right]\left[\mathcal{D} \theta_{v, I}\right]\right) \exp \left[i \int_{M^{d+1}}\left(\sum_{I=1}^{N} \frac{\chi_{I}}{2}\left(d \theta_{s, I}+d \theta_{v, I}\right) \wedge \star\left(d \theta_{s, I}+d \theta_{v, I}\right)+\frac{2}{2 \pi} \sum_{I=1}^{N} B_{I} d\left(d \theta_{v, I}\right)\right)\right] \\
& \cdot \omega_{d+1}\left(\left\{d \theta_{v, I}\right\}\right) .
\end{aligned}
$$

where $\omega_{d+1}\left(\left\{d \theta_{v, I}\right\}\right)$ is mapped to a multikink Berry phase topological term $\exp \left[i \int_{M^{d+1}} \#\left(d \theta_{v, 1}\right) \wedge \cdots \wedge\left(d \theta_{v, N}\right)\right]$ $[32,33]$.
We replace and redefine a derivative on the right-hand side by substituting $C=d \theta_{v}\left(\right.$ or $\left.C_{\mu}=\partial_{\mu} \theta_{v}\right):^{14}$

$$
\begin{aligned}
D_{\mu}^{c, \operatorname{Im}} \Phi & \rightarrow D_{\mu}^{d \theta_{v}, \operatorname{Im}} \Phi:=\partial_{\mu} \Phi-i g_{c}\left(\partial_{\mu} \theta_{v}\right) \Phi_{\operatorname{Im}}, \\
D_{\mu}^{A, c, \operatorname{Im}} \Phi \rightarrow D_{\mu}^{A, d \theta_{v}, \operatorname{Im}} \Phi: & :=D_{\mu}^{A} \Phi-i g_{c}\left(\partial_{\mu} \theta_{v}\right) \Phi_{\operatorname{Im}}=\left(\partial_{\mu}-i g A_{\mu}\right) \Phi-i g_{c}\left(\partial_{\mu} \theta_{v}\right) \Phi_{\operatorname{Im}}, \\
D_{\mu}^{c} A_{v, i_{2}, \ldots, i_{m}} \rightarrow D_{\mu}^{d \theta_{v}} A_{v, i_{2}, \ldots, i_{m}}: & =\left[\partial_{\mu}-i g_{c}\left(\partial_{\mu} \theta_{v}\right)\right] A_{v, i_{2}, \ldots, i_{m}}, \\
\hat{F}_{\mu, v, i_{2}, \ldots, i_{m}}^{c} \rightarrow \hat{F}_{\mu, v, i_{2}, \ldots, i_{m}}^{d \theta_{v}}: & =D_{\mu}^{d \theta_{v}} A_{v, i_{2}, \ldots, i_{m}}-D_{v}^{d \theta_{v}} A_{\mu, i_{2}, \ldots, i_{m}} .
\end{aligned}
$$

We can either include or omit the $I$ index for these operators.

By combining two kinds of vortex degrees of freedom from the vortex 1 of $\phi$ and the vortex 2 of $\theta$, we can rewrite Eq. (2.96) into a sigma-model-like expression for a non-Abelian gauged fractonic matter theory:

$$
\begin{aligned}
\mathbf{Z}_{\mathrm{rk}-(m+1)-\mathrm{NAb}-\mathrm{vortex}}^{\text {sigma model }}= & \int\left(\prod_{I=1}^{N}\left[\mathcal{D} A_{I}\right]\left[\mathcal{D} B_{I}\right]\left[\mathcal{D} \theta_{s, I}\right]\left[\mathcal{D} \theta_{v, I}\right]\left[\mathcal{D} \rho_{I}\right]\left[\mathcal{D} \phi_{I}\right]\right) \exp \left\{i \int _ { M ^ { d + 1 } } \sum _ { I = 1 } ^ { N } \left[d ^ { d + 1 } x \left(\left|\hat{F}_{\mu, v, i_{2}, \ldots, i_{m}}^{d \theta_{v}, I}\right|^{2}+\sum_{I=1}^{N} \frac{\chi_{I}}{2} \mid d \theta_{s, I}\right.\right.\right. \\
& +\left.d \theta_{v, I}\right|^{2}+D_{i_{1}, \ldots, i_{m}}^{A, d \theta_{v}, \operatorname{Im}}\left[\left\{\sqrt{\rho_{I}} \exp \left(i \phi_{I}\right)\right\}\right] D_{A, d \theta_{v}, \operatorname{Im}}^{\dagger i_{1}, \ldots, i_{m}}\left[\left\{\sqrt{\rho_{I}} \exp \left(-i \phi_{I}\right)\right\}\right]+D_{i_{1}, \ldots, i_{m}}^{\dagger A, d \theta_{v}, \operatorname{Im}}\left[\left\{\sqrt{\rho_{I}} \exp \left(i \phi_{I}\right)\right\}\right] D_{A, d \theta_{v}, \operatorname{Im}}^{i_{1}, \ldots, i_{m}} \\
& \left.\left.\left.\times\left[\left\{\sqrt{\rho_{I}} \exp \left(-i \phi_{I}\right)\right\}\right]+V\left(\left\{\left|\rho_{I}\right|\right\}\right)\right)+\frac{2}{2 \pi} B_{I} d\left(d \theta_{v, I}\right)\right]\right\} \cdot \omega_{d+1}\left(\left\{d \theta_{v, I}\right\}\right)
\end{aligned}
$$

Here we have substituted Eq. (3.1), Eq. (3.11), and $\left|\Phi_{I}\right|^{2}=$ $\left|\rho_{I}\right|$. Equation (3.17) is the most generic form of a sigma model: a part of its phase diagram gives rise to the TQFT

as a 1-form gauge field, to describe the vortex core. The partition function in the disordered state away from the superfluid now becomes that of a gapped insulator (for $N=1$ ) or topologically ordered state with a topological level- $N$ BF action as a $\mathbb{Z}_{N}$-gauge theory:

$$
\begin{aligned}
\mathbf{Z} & =\int[\mathcal{D} b][\mathcal{D} a] \exp \left(\frac{i}{2 \pi} \int B \wedge d C\right) \\
& =\int[\mathcal{D} b][\mathcal{D} a] \exp \left(i \int \frac{d^{d} x}{2 \pi(d-2) !} \epsilon^{\mu \mu_{2} \cdots \mu_{d}} B_{\mu_{2} \mu_{3} \ldots} \partial_{\mu_{d-1}} C_{\mu_{d}}\right) .
\end{aligned}
$$

${ }^{14} \mathrm{We}$ also have the gauge transformation descended from a 1-form $C$ gauge field,

$$
\begin{aligned}
\partial_{v} \theta_{v, I} & \rightarrow \partial_{\nu} \theta_{v, I}+\frac{1}{g_{c}} \partial_{\nu} \gamma_{c, I}(x), \\
\theta_{v, I} & \rightarrow \theta_{v, I}+\frac{1}{g_{c}} \gamma_{c, I}(x) .
\end{aligned}
$$

(when the $\theta_{v, I}$ vortices disordered), while the other part of its phase diagram gives rise to the spontaneously symmetry breaking superfluidlike phases. We emphasize that $d\left(d \theta_{v, I}\right)=$ $d^{2} \theta_{v, I}$ is nonzero and can be related to a quantized number such as a winding number at the core of the vortex field; see Sec. IV.

Here $B$ is only a Lagrange multiplier. Here we also have not yet replaced the symmetric tensor gauge field $A$ with any kinds of vortex degrees of freedom in Eq. (3.17). As some readers may wonder, it is tempting to ask whether the gauge field $A$ can be "dualized" into some new vortex degrees of freedom. However, we will not attempt to attack this issue and leave this as an open question for future work.

\section{CAUCHY-RIEMANN RELATION, WINDING NUMBER, AND TOPOLOGICAL DEGREE THEORY}

Here we derive a relation used in the previous section, relating the vortex degrees of freedom to a winding number, via the Cauchy-Riemann relation and topological degree theory, at least for the two-dimensional space mapping to a deformed 
$S^{1}$-circle as a target space. On the complex plane

$$
z:=x+i y=r \exp (i \varphi),
$$

we define the Hodge star operator $\star$ (for the differential form, this is the Hodge dual) on the 1-form as

$$
\star(f d x+g d y)=(-g d x+f d y),
$$

for some generic functions $f$ and $g$.

Then we have $\star d z=-i d z$ with $d z=d x+i d y$. We can compute that

$$
d \star d f=(\Delta f)(d x \wedge d y),
$$

with the Laplacian or Laplace operator $\Delta \equiv \nabla^{2}$.

If $f=u+i v$ is holomorphic dependent on $z$ independent of $\bar{z}$ (namely the Cauchy-Riemann equation), $d f=f^{\prime} d z$ and also $i \star d f=f^{\prime} d z$ with $f^{\prime}=\frac{d f}{d z}$, so we see that $d v=\star d u$.

Taking $f=\log z=\log [r \exp (i \varphi)]=\log r+i \varphi$, then $u=$ $\log r$ and $v=\varphi$, where $z=r e^{i \varphi}$ is the polar coordinate, and then we have

$$
d d \varphi=d d v=d \star d u=(\Delta \log r)(d x \wedge d y)=2 \pi \delta_{0},
$$

where

$$
\delta_{0} \equiv \delta(\mathbf{r})(d x \wedge d y)
$$

is the delta function at the origin 0 of the polar coordinate. ${ }^{15}$ Hence we derive that

$$
\frac{1}{2 \pi} d d \varphi=\delta_{0} .
$$

For a general $S^{1}$ valued function $\phi$ defined outside a singular point $p$, we may assume $p=0$ at the origin (without loss of generality).

There are applications to two types of vortices:

(1) For the fractonic phase field $\phi$ : We can always write the phase of the fractonic matter field in Eq. (3.1) as

$$
\phi:=\phi_{s}+\phi_{v}:=\phi_{s}+n \varphi,
$$

where $\phi_{s}$ captures the smooth $(s)$ part and $\phi_{v}$ captures the singular vortex $(v)$ part, while $n$ is the winding number. In

\footnotetext{
${ }^{15}$ Here we use facts about the fundamental solutions of Laplace's or Poisson's equations by solving the source of the Dirac delta function $\delta(r)$; see for example [36]. The volume of $D$-dimensional ball $B^{D}$ is $V_{D} \equiv \frac{\pi^{D / 2}}{\Gamma(D / 2+1)}$, where the gamma function obeys $\Gamma(n+1)=n \Gamma(n)$ for general $n \in \mathbb{C}, \Gamma(1 / 2)=\sqrt{\pi}$, while $\Gamma(n)=(n-1)$ ! for any positive integer $n$. The area of the boundary of the $D$-dimensional ball $B^{D}$ is the hypervolume of the $(D-1)$-dimensional sphere, denoted as $A_{B^{D}} \equiv A_{S^{D-1}}=D V_{D} \equiv \frac{D \pi^{D / 2}}{\Gamma(D / 2+1)}=\frac{2 \pi^{D / 2}}{\Gamma(D / 2)}$. Given the vector $\mathbf{r} \in \mathbb{R}^{D}$ and the radial $r=|\mathbf{r}|$, then we have the following properties for Laplacian $\Delta=\nabla^{2}$ :

$$
\begin{aligned}
D & =2, \quad \nabla^{2}(\ln (r))=2 \pi \delta^{(2)}(\mathbf{r}), \\
D & =3, \quad \nabla^{2}\left(r^{-1}\right)=-4 \pi \delta^{(3)}(\mathbf{r}), \\
& \vdots \\
D & \geqslant 3, \quad \nabla^{2}\left(r^{-(D-2)}\right)=-(D-2) A_{S^{D-1}} \delta^{(D)}(\mathbf{r}) .
\end{aligned}
$$
}

In general $\delta_{0} \equiv \delta(\mathbf{r})\left(\operatorname{vol}_{D}\right)=\delta(\mathbf{r})\left(d x_{1} \wedge \cdots \wedge d x_{D}\right)$. terms of the degree theory, we only focus on $\Sigma^{2} \rightarrow S^{1}$, specifically here we consider $\Sigma^{2}=\mathbb{R}^{2}-\{0\}$ as a punctured 2-plane mapping to a circle $S^{1}$ [or the U(1) target space]. We can possibly generalize this result to other target spaces. The first term $\phi_{s}$ that extends smoothly over 0 is known as the smooth fluctuation. Then using the previous result we see that

$$
\begin{aligned}
\frac{1}{2 \pi} d d \phi & =\frac{1}{2 \pi} d d\left(\phi_{s}+\phi_{v}\right)=\frac{1}{2 \pi} d\left(\phi_{v}\right) \\
& =n \frac{1}{2 \pi} d d(\varphi)=n \delta_{0} \quad \text { with } n \in \mathbb{Z} .
\end{aligned}
$$

Thus, importantly, we can identify the solution of vortex core equation $\frac{1}{2 \pi} d^{2} \phi_{v}=n \delta_{0}$ with $n \in \mathbb{Z}$ as the winding number.

(2) For the $\mathbb{Z}_{2}^{C}$-gauge phase field $\theta$ : Similarly, for the vortex associated with the $C$ gauge field,

$$
\begin{gathered}
\frac{1}{2 \pi} d d \theta=\frac{1}{2 \pi} d d\left(\theta_{s}+\theta_{v}\right)=\frac{1}{2 \pi} d d\left(\theta_{v}\right)=n_{\theta_{v}} \delta_{0} \quad \text { with } \\
n_{\theta_{v}} \in \mathbb{Z} .
\end{gathered}
$$

So if the Cauchy-Riemann relation can be applied, we can rewrite

$$
\frac{2}{2 \pi} B_{I} d\left(d \theta_{v, I}\right)=B_{I} n_{\theta_{v}} \delta_{0}
$$

to a term associated with the winding number $n_{\theta_{v}}$. We can adjust $\delta_{0}$ from the location of the origin to other points $p$ as $\delta_{p}$ for all the above discussions to indicate the locations of vortices at points $p$.

\section{CONCLUSIONS}

We have proposed a systematic framework to obtain a family of non-Abelian gauged fractonic matter field theories in Sec. II. We have derived a family of sigma models with two types of vortices in Sec. III that can interplay and transition between the disordered phase (with higher-rank tensor non-Abelian gauge theories coupled to fractonic matter) and the ordered phase (with superfluids and vortex excitations) of sigma models. We formulate two types of vortices: one is associated to the fractonic matter fields $[d(d \phi)]$, the other is associated to the 1-form $C$ gauge field $\left[d\left(d \theta_{v}\right)\right]$. These two types of vortices mutually interact noncommutatively when they communicate via the higher-rank tensor gauge field $A$ as a propagator. We apply the Cauchy-Riemann relation and topological degree theory to capture the winding number for the two types of vortices in Sec. IV.

Here we make some extended comments and also list some open questions.

(1) Reduction to the Abelian case: When the 1-form gauge field $C=0$, we reduce to the Abelian tensor gauged matter theory. If we further turn off the tensor gauge field $A$, then our sigma model should reduce to a simplified special case of the Abelian fractonic superfluid models in Ref. [30]. When we turn on the gauge field $C$, there are nontrivial couplings between matter sectors and antimatter sectors, because the particle-hole symmetries are dynamically gauged.

(2) Our sigma model Eq. (3.17) contains the target space with radius size $\sqrt{\rho}$. 
If the $\sqrt{\rho}$ is fixed, then we have a fixed radius $S^{1}$ target space, and we only have the fluctuation around the $\phi$ fields (e.g., Goldstone modes, superfluid, or vortices).

If the $\sqrt{\rho}$ also fluctuates, the volume and radius size of the sigma model target space change, so we have more interesting dynamics for the sigma model. Its dynamics and low energy fates are interesting, but perplexing and challenging, and are important questions for the future.

(3) We had mentioned that two types of vortices mutually interact via tensor gauge field $A$, causing some kind of nonAbelian vortex behavior altogether. Moreover, it is tempting to know whether we can also dualize tensor gauge field $A$ to represent the third type of vortex from $A$.

(4) Our formulation of sigma models may have applications to superfluids, supersolids, the quantum melting transition, and elasticity studied in the recent fracton literature (for selected references and quantum crystal disclinations and dislocations, see [37-42] and citations therein).

(5) In the present literature, there are three different routes to obtain non-Abelian fracton orders:

(a) Gauge the charge conjugation (i.e., particle-hole) $\mathbb{Z}_{2}^{C}$ symmetry and $\mathrm{U}(1)_{\text {poly }}$ polynomial symmetry $[4,6]$.

(b) Gauge the permutation symmetry of $N$-layer systems $[19,20]$.

(c) Couple to non-Abelian TQFT/topological order $[18,43,44]$ and $[4,6]$.

Given $N$-layer systems, there is a larger non-Abelian group structure that we can explore. In the previous work $[4,6]$ and our present work, we focus on the finite Abelian group $\left(\mathbb{Z}_{2}^{C}\right)^{N}$ by gauging $N$-layer of particle-hole symmetries, and consider a non-Abelian gauge structure: $\mathrm{U}(1)_{\text {poly }} \rtimes\left(\mathbb{Z}_{2}^{C}\right)^{N}$. In fact, a natural larger group includes also the $\mathrm{S}_{N}$ permutation symmetry group of $N$ layers $[19,20]$. The $\left(\mathbb{Z}_{2}^{C}\right)^{N}$ and $\mathrm{S}_{N}$ form a short exact sequence via a group extension:

$$
1 \rightarrow\left(\mathbb{Z}_{2}^{C}\right)^{N} \rightarrow G_{\mathrm{nAb}} \rightarrow \mathrm{S}_{N} \rightarrow 1 .
$$

The $G_{\mathrm{nAb}}$ is related to the hyperoctahedral group in mathematics. An overall larger non-Abelian group structure including both $G_{\mathrm{nAb}}$ and $\mathrm{U}(1)_{\text {poly }}$, that mutually are noncommutative, possibly can be studied also via field theories or lattice models in the future.

(6) Reference [6] points out possible proper tools for studying these field theories, including algebraic variety and affine-geometry/manifold. One motivation is to explore these theories and more general models on general affine manifolds beyond the Euclidean spacetime. This is left for future work.

(7) Quantization and quantum path integral: Although we write down schematic path integral forms, our analysis is mostly based on equations of motion (EOM) and semiclassical or mean-field analyses. The most challenging question may be to explore the full quantum nature of the path integral we proposed, or study the quantization of these field theories. However, since we do have a quantum mechanical definition of the theory on a regularized lattice with an energy cutoff,

(a) the U(1) tensor field theory on the lattice ([13] and $[1,45])$, and

(b) the topological gauge theory from group cohomology (of Dijkgraaf-Witten type) on the lattice [44,46,47], we believe that our field theories (as the interplay between the two models) should be a promising quantum theory, and quantum field theories can be made to be mathematically rigorously well defined.

\section{ACKNOWLEDGMENTS}

J.W. thanks Kai Xu and John Loftin for enlightening conversations. The method of deriving a family of sigma model theories partially originated from [32] and an unpublished work of 2015-2016. J.W. thanks Abhinav Prem and Yunqin Zheng for feedback, and the attendees of the Quantum Matter in Math and Physics workshop [48]. J.W. was supported by NSF Grant No. PHY-1606531 and Institute for Advanced Study. This work is also supported by NSF Grant No. DMS1607871, "Analysis, Geometry and Mathematical Physics" and by the Center for Mathematical Sciences and Applications at Harvard University.
[1] R. M. Nandkishore and M. Hermele, Fractons, Annu. Rev. Condensed Matter Phys. 10, 295 (2019)..

[2] M. Pretko, The fracton gauge principle, Phys. Rev. B 98, 115134 (2018).

[3] N. Seiberg, Field theories with a vector global symmetry, SciPost Phys. 8, 050 (2020).

[4] J. Wang and K. Xu, Higher-rank tensor field theory of nonAbelian fracton and embeddon, arXiv:1909.13879.

[5] A. Gromov, Towards classification of fracton phases: The multipole algebra, Phys. Rev. X 9, 031035 (2019).

[6] J. Wang, K. Xu, and S.-T. Yau, Higher-rank tensor non-Abelian field theory: Higher-moment or subdimensional polynomial global symmetry, algebraic variety, Noether's theorem, and gauge, arXiv:1911.01804.

[7] T. Griffin, K. T. Grosvenor, P. Horava, and Z. Yan, Scalar field theories with polynomial shift symmetries, Commun. Math. Phys. 340, 985 (2015).
[8] C. Chamon, Quantum Glassiness in Strongly Correlated Clean Systems: An Example of Topological Overprotection, Phys. Rev. Lett. 94, 040402 (2005).

[9] C. Castelnovo and C. Chamon, Topological quantum glassiness, Philos. Mag. 92, 304 (2012).

[10] E. Schrödinger, An undulatory theory of the mechanics of atoms and molecules, Phys. Rev. 28, 1049 (1926).

[11] O. Klein, Quantentheorie und fünfdimensionale Relativitätstheorie, Z. Phys. 37, 895 (1926).

[12] W. Gordon, Der Comptoneffekt nach der Schrödingerschen Theorie, Z. Phys. 40, 117 (1926).

[13] A. Rasmussen, Y.-Z. You, and C. Xu, Stable gapless Bose liquid phases without any symmetry, arXiv:1601.08235.

[14] M. Pretko, Subdimensional particle structure of higher rank U(1) spin liquids, Phys. Rev. B 95, 115139 (2017).

[15] M. Pretko, Generalized electromagnetism of subdimensional particles: A spin liquid Story, Phys. Rev. B 96, 035119 (2017). 
[16] M. Pretko, Higher-spin Witten effect and two-dimensional fracton phases, Phys. Rev. B 96, 125151 (2017).

[17] K. Slagle, A. Prem, and M. Pretko, Symmetric tensor gauge theories on curved spaces, Ann. Phys. (NY) 410, 167910 (2019).

[18] A. Prem, S.-J. Huang, H. Song, and M. Hermele, Cage-Net Fracton Models, Phys. Rev. X 9, 021010 (2019).

[19] D. Bulmash and M. Barkeshli, Gauging fractons: Immobile non-Abelian quasiparticles, fractals, and position-dependent degeneracies, Phys. Rev. B 100, 155146 (2019).

[20] A. Prem and D. J. Williamson, Gauging permutation symmetries as a route to non-Abelian fractons, SciPost Phys. 7, 068 (2019).

[21] R. Dijkgraaf and E. Witten, Topological gauge theories and group cohomology, Commun. Math. Phys. 129, 393 (1990).

[22] J. Wang, X.-G. Wen, and S.-T. Yau, Quantum statistics and spacetime surgery, Phys. Lett. B 807, 135516 (2020).

[23] P. Putrov, J. Wang, and S.-T. Yau, Braiding statistics and link invariants of bosonic/fermionic topological quantum matter in 2+1 and 3+1 dimensions, Ann. Phys. (NY) 384, 254 (2017).

[24] J. Wang, K. Ohmori, P. Putrov, Y. Zheng, Z. Wan, M. Guo et al., Tunneling topological vacua via extended operators: (Spin-)TQFT spectra and boundary deconfinement in various dimensions, Prog. Theor. Exp. Phys. 2018, 053 A01 (2018).

[25] J. Wang, X.-G. Wen, and S.-T. Yau, Quantum statistics and spacetime topology: Quantum surgery formulas, Ann. Phys. (NY) 409, 167904 (2019).

[26] V. L. Ginzburg and L. D. Landau, On the theory of superconductivity, Zh. Eksp. Teor. Fiz. 20, 1064 (1950).

[27] M. P. A. Fisher and D. H. Lee, Correspondence between twodimensional bosons and a bulk superconductor in a magnetic field, Phys. Rev. B 39, 2756 (1989).

[28] C. Dasgupta and B. I. Halperin, Phase Transition in a Lattice Model of Superconductivity, Phys. Rev. Lett. 47, 1556 (1981).

[29] D. R. Nelson, Vortex Entanglement in High- $T_{c}$ Superconductors, Phys. Rev. Lett. 60, 1973 (1988).

[30] J.-K. Yuan, S. A. Chen, and P. Ye, Fractonic superfluids, topological vortices, and quantum fluctuations, Phys. Rev. Research 2, 023267 (2020).

[31] C. N. Yang and R. L. Mills, Conservation of Isotopic Spin and Isotopic Gauge Invariance, Phys. Rev. 96, 191 (1954).

[32] Z.-C. Gu, J. C. Wang, and X.-G. Wen, Multikink topological terms and charge-binding domain-wall condensation induced symmetry-protected topological states: Beyond ChernSimons/BF theory, Phys. Rev. B 93, 115136 (2016).
[33] P. Ye and Z.-C. Gu, Topological quantum field theory of threedimensional bosonic Abelian-symmetry-protected topological phases, Phys. Rev. B 93, 205157 (2016).

[34] J. Hubbard, Calculation of Partition Functions, Phys. Rev. Lett. 3, 77 (1959).

[35] P. Ye and Z.-C. Gu, Vortex-Line Condensation in Three Dimensions: A Physical Mechanism for Bosonic Topological Insulators, Phys. Rev. X 5, 021029 (2015).

[36] L. C. Evans, Partial Differential Equations (American Mathematical Society, Providence, RI, 2010).

[37] M. Pretko and L. Radzihovsky, FractonElasticity Duality, Phys. Rev. Lett. 120, 195301 (2018).

[38] M. Pretko and L. Radzihovsky, Symmetry-Enriched Fracton Phases from Supersolid Duality, Phys. Rev. Lett. 121, 235301 (2018).

[39] L. Radzihovsky and M. Hermele, Fractons from vector gauge theory, Phys. Rev. Lett. 124, 050402 (2020).

[40] M. Pretko, Z. Zhai, and L. Radzihovsky, Crystal-to-fracton tensor gauge theory dualities, Phys. Rev. B 100, 134113 (2019).

[41] A. Gromov and P. Surowka, On duality between Cosserat elasticity and fractons, SciPost Phys. 8, 065 (2020).

[42] Y. You, Z. Bi, and M. Pretko, Emergent fractons and algebraic quantum liquid from plaquette melting transitions, Phys. Rev. Research 2, 013162 (2020).

[43] S. Vijay and L. Fu, A Generalization of non-Abelian anyons in three dimensions, arXiv:1706.07070.

[44] H. Song, A. Prem, S.-J. Huang, and M. A. Martin-Delgado, Twisted fracton models in three dimensions, Phys. Rev. B 99, 155118 (2019).

[45] M. Pretko, X. Chen, and Y. You, Fracton phases of matter, Int J. Mod. Phys. A 35, 2030003 (2020).

[46] Y. Hu, Y. Wan, and Y.-S. Wu, Twisted quantum double model of topological phases in two dimensions, Phys. Rev. B 87, 125114 (2013).

[47] Y. Wan, J. C. Wang, and H. He, Twisted gauge theory model of topological phases in three dimensions, Phys. Rev. B 92, 045101 (2015).

[48] J. C. Wang, Higher-rank tensor non-Abelian gauge field theory of fracton and embeddon, in Quantum Matter workshop, Quantum Matter in Mathematics and Physics at Harvard CMSA, December 3, 2019 https://www.youtube.com/watch?v= 77vkcOrvW8k (unpublished). 